\title{
Targeting methyltransferase PRMT5 eliminates leukemia stem cells in chronic myelogenous leukemia
}

\author{
Yanli Jin, ${ }^{1}$ Jingfeng Zhou, ${ }^{1}$ Fang Xu, ${ }^{1}$ Bei Jin, ${ }^{1}$ Lijing Cui, ${ }^{2}$ Yun Wang, ${ }^{1}$ Xin Du, ${ }^{3}$ Juan Li, ${ }^{4}$ Peng Li, ${ }^{5}$ Ruibao Ren, ${ }^{6}$ and Jingxuan Pan ${ }^{1,7}$ \\ IJinan University Institute of Tumor Pharmacology, and State Key Laboratory of Ophthalmology, Zhongshan Ophthalmic Center, Sun Yat-sen University, Guangzhou, China. ²Department of Pathophysiology, \\ Zhongshan School of Medicine, Sun Yat-sen University, Guangzhou, China. ${ }^{3}$ Department of Hematology, Guangdong General Hospital/Guangdong Academy of Medical Sciences, Guangzhou, China. \\ ${ }^{4}$ Department of Hematology, The First Affiliated Hospital, Sun Yat-sen University, Guangzhou, China. ${ }^{5}$ Guangzhou Institute of Biomedicine and Health, Chinese Academy of Sciences, Guangzhou, China. \\ ${ }^{6}$ State Key Laboratory for Medical Genomics and Shanghai Institute of Hematology, Ruijin Hospital, Shanghai, China. 'Collaborative Innovation Center for Cancer Medicine, State Key Laboratory of Oncology \\ in South China, Sun Yat-sen University Cancer Center, Guangzhou, China.
}

Imatinib-insensitive leukemia stem cells (LSCs) are believed to be responsible for resistance to BCR-ABL tyrosine kinase inhibitors and relapse of chronic myelogenous leukemia (CML). Identifying therapeutic targets to eradicate CML LSCs may be a strategy to cure CML. In the present study, we discovered a positive feedback loop between BCR-ABL and protein arginine methyltransferase 5 (PRMT5) in CML cells. Overexpression of PRMT5 was observed in human CML LSCs. Silencing PRMT5 with shRNA or blocking PRMT5 methyltransferase activity with the small-molecule inhibitor PJ-68 reduced survival, serial replating capacity, and long-term culture-initiating cells (LTC-ICs) in LSCs from CML patients. Further, PRMT5 knockdown or PJ-68 treatment dramatically prolonged survival in a murine model of retroviral BCR-ABL-driven CML and impaired the in vivo self-renewal capacity of transplanted CML LSCs. PJ-68 also inhibited long-term engraftment of human CML CD34+ cells in immunodeficient mice. Moreover, inhibition of PRMT5 abrogated the Wnt/ $\beta$-catenin pathway in CML CD34+ cells by depleting dishevelled homolog 3 (DVL3). This study suggests that epigenetic methylation modification on histone protein arginine residues is a regulatory mechanism to control self-renewal of LSCs and indicates that PRMT5 may represent a potential therapeutic target against LSCs.

\section{Introduction}

Chronic myelogenous leukemia $(\mathrm{CML})$ is a disease of hematopoietic stem cells (HSCs) malignantly transformed by the formation of the Philadelphia chromosome (i.e., $B C R-A B L$ fusion gene) due to reciprocal chromosomal translocation $\mathrm{t}(9,22)$ (q34;q11) (1). CML is characterized by malignant expansion of myeloid leukemia cells in bone marrow (BM) and peripheral blood circulation (2). Patients with CML usually experience 3 clinical phases: chronic phase (CP), when BCR-ABL is usually the only driver of the disease; accelerated phase (AP); and blast phase/crisis (BP), when additional oncogenic factors are involved and the disease may clinically resemble acute leukemia (1). Consequently, patients with CP-CML respond well to the tyrosine kinase inhibitor (TKI) imatinib mesylate (IM), whereas patients with AP- and BP-CML usually show IM resistance and CML relapse $(2,3)$.

Acquired resistance to IM accounts for approximately $40 \%$ $50 \%$ of resistance cases and is mainly due to mutations in the $B C R$ $A B L$ gene (e.g., T315I, G250E, Q252H, Y253H, and E255K/V) (3, $4)$. The drug resistance caused by most of the point mutations in $B C R-A B L$ may be conquered with the second generation (e.g., nilotinib and dasatinib) and the third generation (e.g., ponatinib) of TKIs $(5,6)$. The evolution of BCR-ABL-independent leukemia clones is the second mechanism to render IM resistance $(3,7)$. Some CML patients show primary resistance to IM. Adult CML

Conflict of interest: The authors have declared that no conflict of interest exists. Submitted: October 26, 2015; Accepted: August 11, 2016.

Reference information: J Clin Invest. 2016;126(10):3961-3980. doi:10.1172/JCI85239. patients in $\mathrm{AP}$ and $\mathrm{BP}$ and $30 \%$ of $\mathrm{BCR}-\mathrm{ABL}^{+}$pediatric patients with acute lymphoblastic leukemia intrinsically fail to respond to the current TKIs, including IM (8). The evolutionary course from $\mathrm{CP}$ to BP usually features additional oncogenic hits, which suggests a switch of the disease driver from BCR-ABL to other drivers or formation of a co-driver complex consisting of multiple oncogenic proteins (9). In such settings, the appearance of BCR-ABLindependent clones may confer resistance to IM and other TKIs (10). The evolutionary pressure to form BCR-ABL-independent leukemia clones may become augmented with long-term IM therapy. Identifying and targeting these additional oncogenic proteins may overcome resistance to IM.

Leukemia stem cells (LSCs) are thought to be an important source of IM resistance, including both primary and acquired resistance (11-13). LSCs possess the properties of rarity, quiescence, self-renewal, and reduced differentiation (11, 12, 14, 15). LSCs maintain their pool size via self-renewal but produce a hierarchy consisting of different stages of leukemic blast cells (10). In addition, the BCR-ABL-independent property of LSCs facilitates their insensitivity to IM (16). This ineffectiveness is supported by long-term follow-up clinical trials of IM in CML showing persistence of LSCs even in patients with undetectable levels of BCR$\mathrm{ABL}$ transcripts during IM therapy and nearly inevitable relapse upon withdrawal of IM (14). Obviously, the cure for CML depends on elimination of the LSCs. Unfortunately, a curative approach to eliminate LSCs and then reconstituting the hematopoietic system with normal HSC transplantation can be performed in only a small number of patients and is accompanied by high risks of morbid- 
A

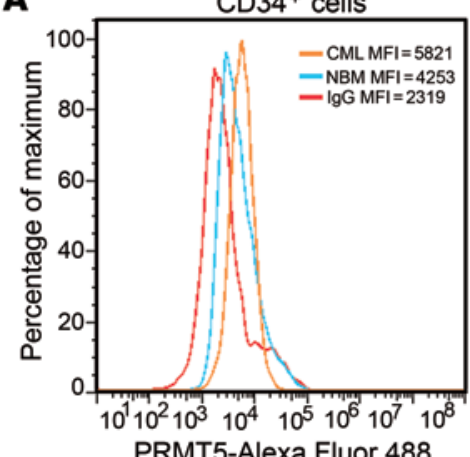

C
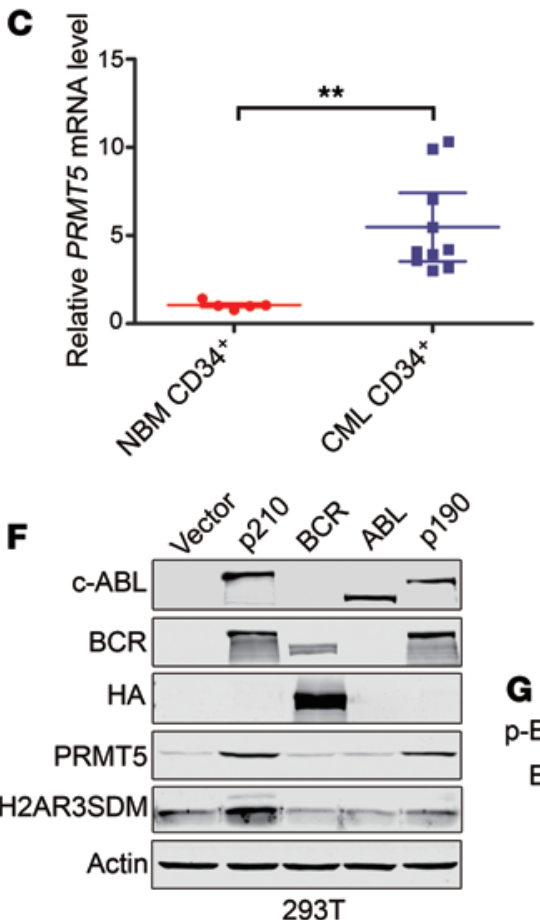

I

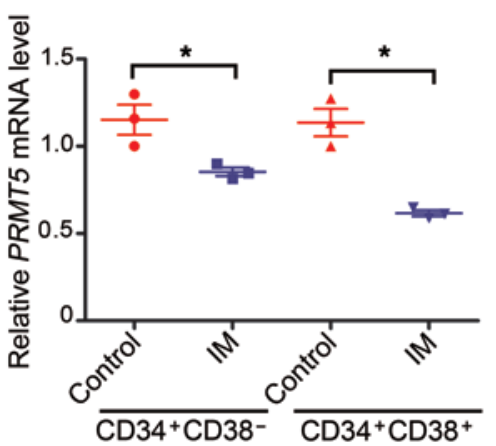

L

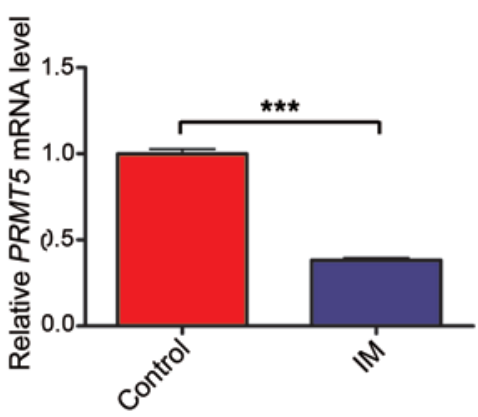

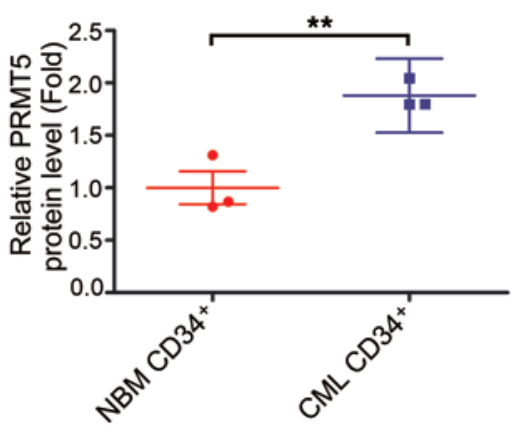

D

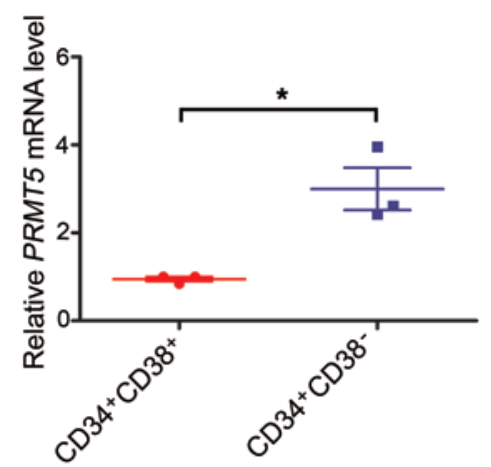

B
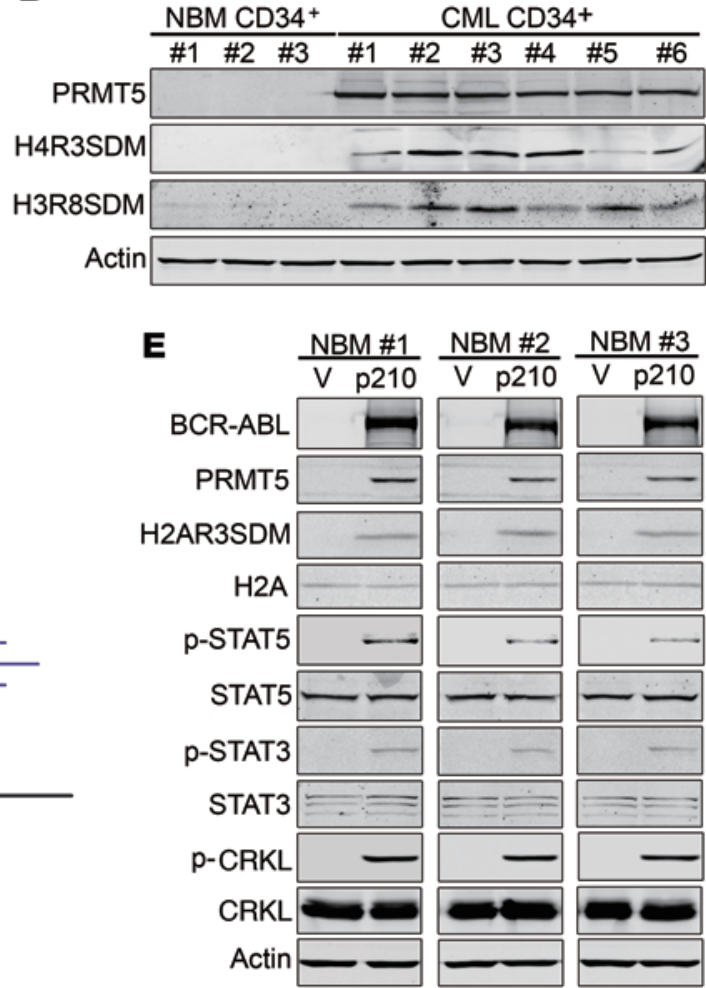

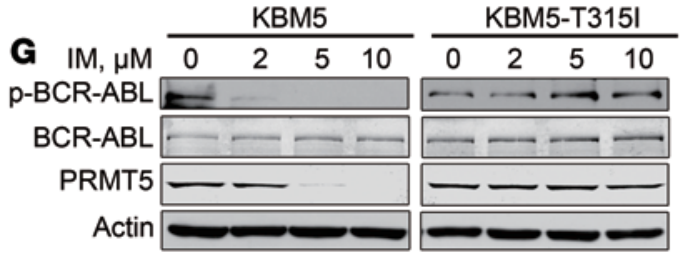

J

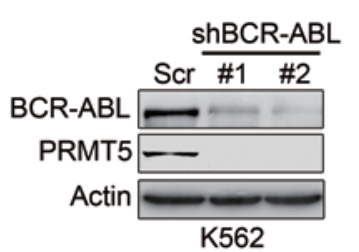

M

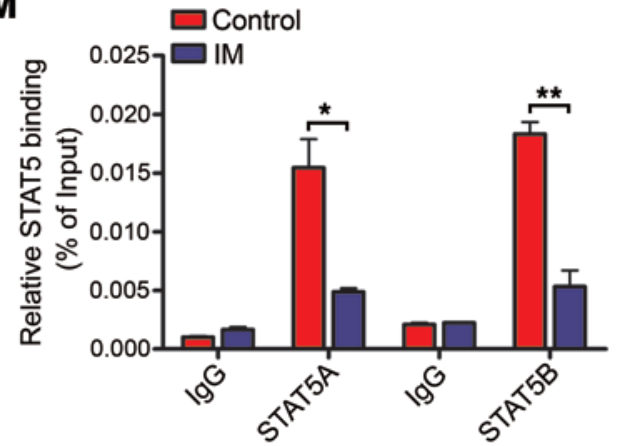

ShBCR-ABL ShBCR-ABL ShBCR-ABL Scr \#1 \#2 Scr \#1 \#2 Scr \#1 \#2

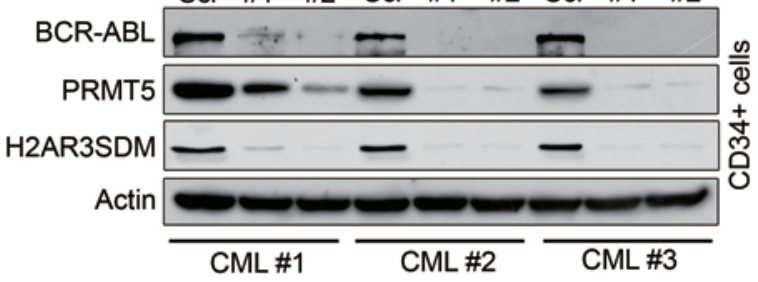

$\mathbf{N}$

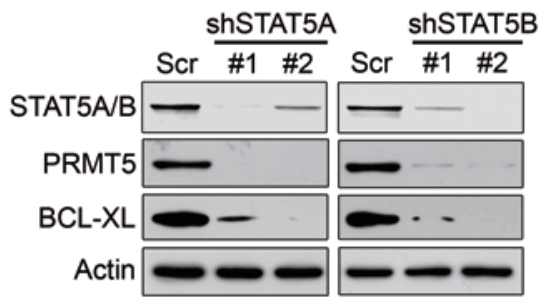


Figure 1. BCR-ABL activates PRMT5 expression in human CD34+ cells. (A) Flow cytometry analysis of intracellular PRMT5 of human CML CD34+ ${ }^{+}$cells relative to NBM CD34+ cells (left). Median fluorescence intensity (MFI) of intracellular PRMT5 protein level in CML $(n=3)$ and NBM $(n=3)$ CD34+ cells (right). (B) The protein levels of PRMT5 and its histone methylation marks H4R3SDM and H3R8SDM were examined by Western blot analysis in CML ( $n=6$ ) and NBM $(n=3)$ CD34+ cells. (C) qRT-PCR of PRMT5 mRNA level in CML $(n=10)$ and NBM $(n=5)$ CD34+ cells. (D) The mRNA level of PRMT5 was higher in CML CD34+CD38- cells than CD34+CD38+ cells $(n=3)$. (E) Western blot analysis of the levels of PRMT5 and its histone methylation mark H2AR3SDM and BCR$A B L$ and its downstream targets STATs and CRKL in NBM CD34+ cells $(n=3)$ transduced with retroviral constructs encoding BCR-ABL (p210) or empty vector (V). (F) Fusion BCR-ABL gene activated PRMT5 expression at the protein level. ( $\mathbf{G}$ and $\mathbf{H}$ ) Inactivation of BCR-ABL activity decreased PRMT5 expression. Uveal melanoma Mel270 cells not harboring BCR-ABL served as a control. (I) The mRNA level of PRMT5 was decreased in CD34+CD38- ${ }^{-}$cells and CD34+CD38 ${ }^{+}$ cells $(n=3)$ from CML patients after IM $(5.0 \mu \mathrm{M})$ treatment for 12 hours. (J and $\mathrm{K}) B C R$ - $A B L$ knockdown by shRNA for 72 hours diminished PRMT5 expression in K562 (J) or primary CML CD34+ cells $(n=3)(\mathbf{K})$. Scr, Scramble shRNA. (L-N) BCR-ABL activated transcription of the PRMT5 gene via STAT5. (L) Inactivation of BCR-ABL decreased PRMT5 mRNA levels. (M) ChIP assay of STAT5 binding to the PRMT5 gene promoter in K562 cells. (N) Protein levels of PRMT5, STAT5A/B and its target protein BCL- $X_{L}$ were determined in K562 cells transduced with lentiviral shRNA against STAT5A or STAT5B for 72 hours. Blot images were derived from samples run on parallel gels. ${ }^{*} P<0.05$, ${ }^{* *} P<0.001$, ${ }^{* *} P<0.0001$, 2-tailed Student's $t$ test.

ity and mortality (10). Therefore, a curative approach for CML should ultimately involve identifying therapeutic targets against LSCs and rationally designing novel small-molecule compounds against specific targets to eradicate LSCs.

LSCs are regulated by multiple mechanisms (17). At the basal level, the fate of LSCs is regulated by survival/apoptosis regulators (e.g., BCL2, BIRC5 [survivin], MCL1) (18). At the second level, the self-renewal capacity of LSCs is regulated by multiple types of proteins: signaling pathways related to HSC development (e.g., Wnt/ $\beta$-catenin, Hedgehog) (13), metabolism regulators (e.g., ALOX5, SCD) (19), transcription factors (e.g., FOXO3, Hif-1 $\alpha$ ), and epigenetic regulators (e.g., SIRT1) (15). At the third level, LSCs are tightly regulated by the malignant hematopoietic microenvironment in CML (20). Targeting epigenetic regulators has recently shown promise for eliminating LSCs while sparing normal HSC cells $(11,16)$. Histone deacetylase (HDAC) inhibitors alone or in combination with TKIs can eliminate quiescent LSCs in both CML and AML $(11,16)$. Besides histone acetylation, methylation of arginine residues of histone proteins is another major mechanism of epigenetic regulation catalyzed by protein arginine transferases and has been linked to leukemogenesis with cancer stem cell-associated (CSC-associated) proteins (21).

Protein arginine methyltransferase 5 (PRMT5), a type II arginine methyltransferase, can catalyze the transfer of a methyl group to 2 of 3 guanidino nitrogen atoms within the arginine molecule in the presence of the cofactor molecule $S$-adenosyl-L-methionine (22). The substrates of PRMT5 include histone and non-histone proteins (e.g., p53). Symmetric dimethylation in histone proteins H4 (S2Me-H4R3) and H3 (S2Me-H3R8) by PRMT5 may remodel the chromatin structure to repress transcription (22). In addition to epigenetic functions, PRMT5 may modify non-histone proteins to regulate various signaling networks (23). PRMT5 is associated with the development of a number of malignancies, such as acute myeloid leukemia, glioblastoma, lymphoma, and lung and ovarian cancer $(23,24)$. In 5-methylthioadenosine phosphorylase-deficient (MTAP-deficient) cancer cells, PRMT5 expression and activity are preferentially required for cell growth, indicating that PRMT5 is an attractive therapeutic target in cancer with these genetic alterations $(25,26)$. PRMT5 is essential for maintaining pluripotency in both embryonic and neural stem cells $(27,28)$. Recently, it has been reported that PRMT5 is critical for sustaining normal hematopoiesis, and its deletion leads to loss of functional hematopoietic stem and progenitor cells (29). However, its role in regulating CSC self-renewal or elimination of CSCs remains unclear.
During a screening for protein targets that regulate LSCs, we discovered a reciprocal positive regulation between PRMT5 and BCR-ABL and the overexpression of PRMT5 in CML LSCs. The objective of this study was to functionally validate PRMT5 as a therapeutic target and to test the effectiveness of specifically targeting PRMT5 by lentiviral shRNA or using a small-molecule inhibitor to eliminate LSCs. Our findings revealed that epigenetic modification by histone arginine methylation is critical for selfrenewal of LSCs, and that therapeutic targeting of PRMT5 holds promise to eliminate LSCs for treatment of CML.

\section{Results}

PRMT5 is highly overexpressed in primary $C D 34^{+}$cells from $C M L$ patients. We first compared PRMT5 expression in CD34 ${ }^{+}$cells from CML patients and normal BM (NBM) from healthy donors. The intracellular level of PRMT5 protein was significantly higher in CML CD $34^{+}$cells than NBM CD $34^{+}$cells (Figure 1A). The protein levels of PRMT5 and its histone methylation marks H4R3SDM and H3R8SDM were significantly increased in CML CD34 ${ }^{+}$cells as determined by Western blot analysis (Figure 1B). The mRNA level of PRMT5 was also greater in CML CD $34^{+}$cells than NBM $\mathrm{CD}^{2} 4^{+}$cells (Figure 1C). Further, the mRNA level of PRMT5 was markedly elevated in CML CD $34^{+} \mathrm{CD} 38^{-}$cells as compared with $\mathrm{CD} 34^{+} \mathrm{CD} 38^{+}$cells (Figure 1D) and normal counterparts (Supplemental Figure 1A, GSE47927; supplemental material available online with this article; doi:10.1172/JCI85239DS1).

PRMT5 expression is increased by BCR-ABL during malignant transformation of human normal $C D 34^{+}$cells. Because CML is believed to be derived from malignant transformation of HSCs triggered by the $B C R-A B L$ fusion oncogene (1), we next determined whether PRMT5 was increased during BCR-ABL-mediated malignant transformation of normal $\mathrm{CD} 34^{+}$cells. Purified NBM CD $34^{+}$cells were transduced with $B C R-A B L$ retroviral MIGR1 (p210) or empty vector (V). The results demonstrated that the protein level of PRMT5 was upregulated, and the BCR-ABL downstream signaling molecules STAT5, STAT3, and CRKL were activated following the transduction of $B C R-A B L$ in NBM CD34 ${ }^{+}$ cells. The activity of PRMT5, as measured by its specific methylation mark, symmetrical dimethylation of histone $\mathrm{H} 2 \mathrm{~A}$ arginine 3 (H2AR3SDM), was also increased by BCR-ABL (Figure 1E).

Activation of PRMT5 transcriptional expression by BCR-ABL fusion gene. To further confirm the positive regulatory effect of BCR-ABL on PRMT5 expression, we transfected 293T cells with vector or pSG5-BCR-ABL (p210) constructs for 48 hours. We 
A

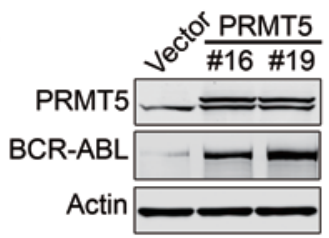

B

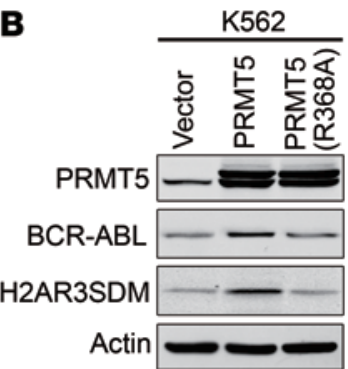

C

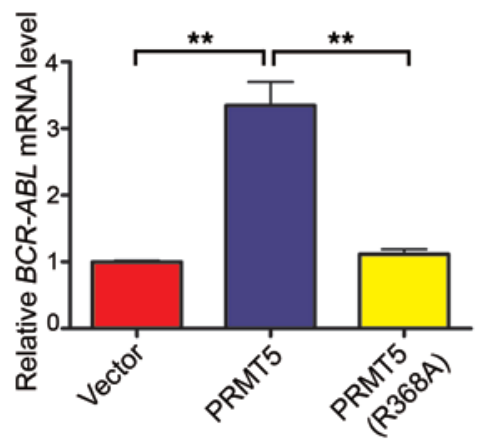

D

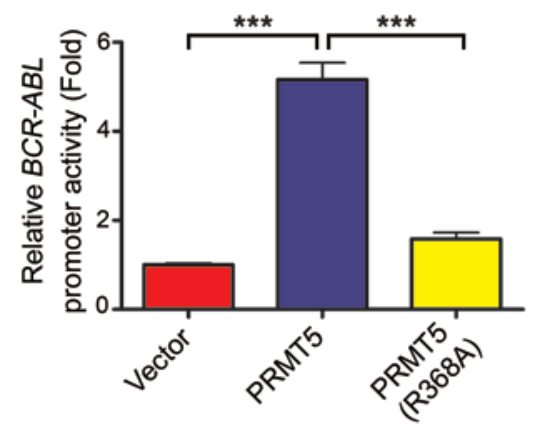

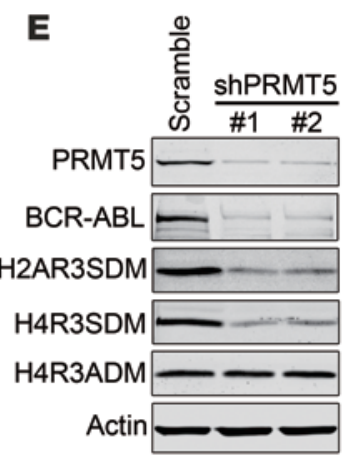

H

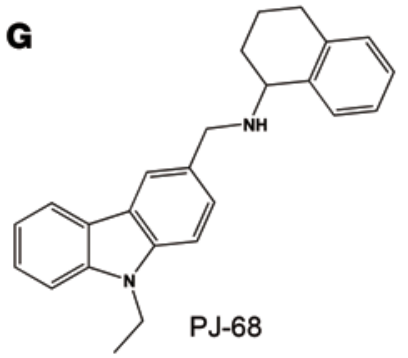

J

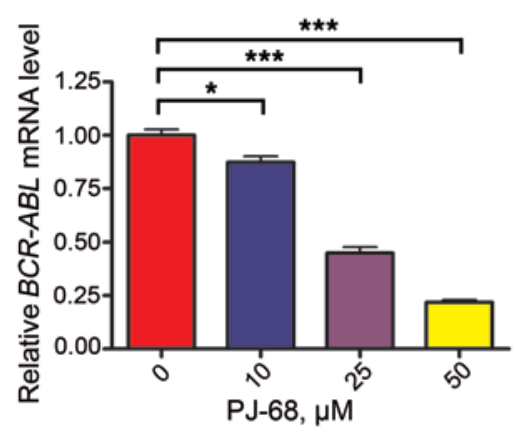

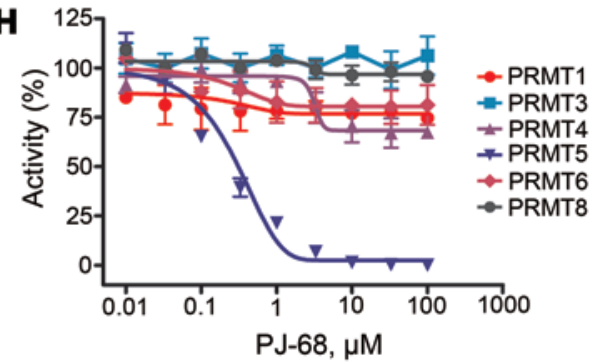

K $\bar{\Phi}$

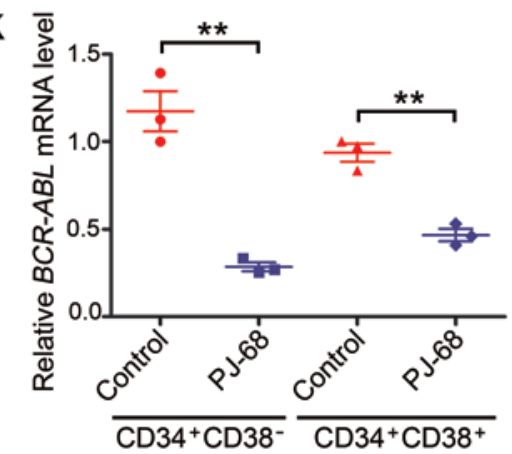

I
F $\bar{\Phi}$
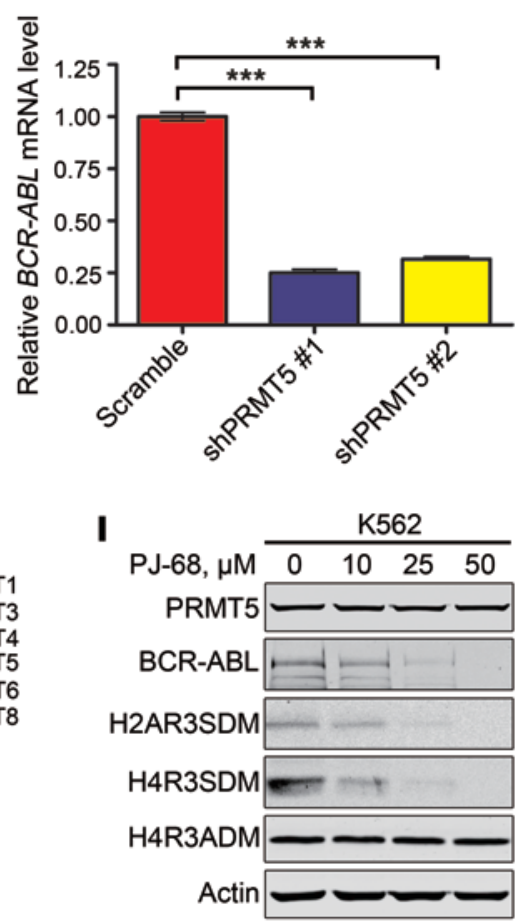

L

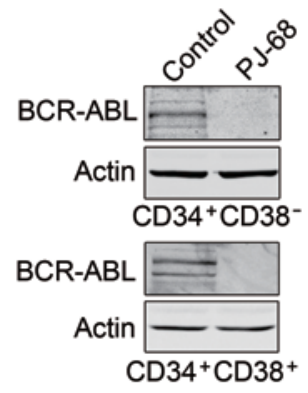

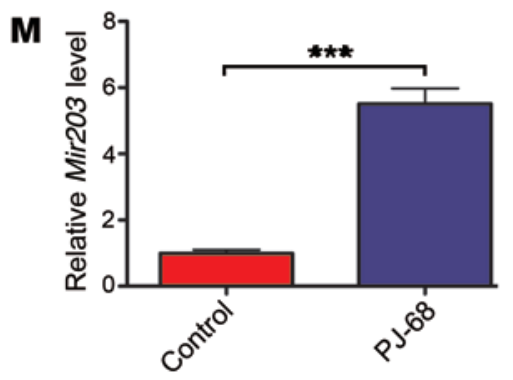
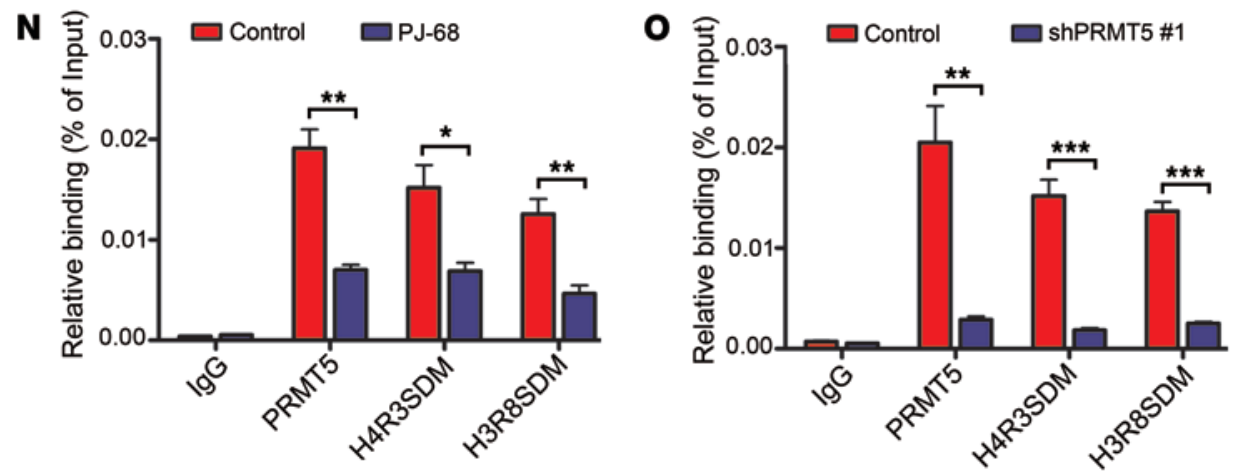
Figure 2. PRMT5 positively regulates BCR-ABL expression in CML cells. (A) Western blot analysis of PRMT5 and BCR-ABL protein levels in K562 cells stably expressing pCMV-2B-Flag (Vector) or pCMV-2B-Flag-PRMT5 (PRMT5) (\#16 and \#19). (B and C) Levels of BCR-ABL protein and mRNA were analyzed by Western blot (B) and qRT-PCR (C) in K562 cells electrotransfected with pCMV-2B-Flag-PRMT5 (PRMT5) or mutant pCMV-2B-Flag-PRMT5 (R368A) [PRMT5 (R368A)]. (D) Luciferase assay of 293T cells transiently cotransfected with the BCR-ABL promoter reporter and PRMT5 or mutant PRMT5 (R368A) constructs for 24 hours. (E) Western blot analysis of BCR-ABL and PRMT5 and its histone methylation marks in K562 with stable PRMT5 knockdown or Scramble control. (F) qRT-PCR analysis of BCR-ABL mRNA levels in $\mathrm{K} 562$ cells with stable PRMT5 knockdown. (C) Chemical structure of PJ-68. (H) Histone methyltransferase activity was measured in the presence of PJ-68 and different PRMT enzymes, including type I PRMT1, $-3,-4,-6$, and -8 and type II PRMT5. PJ-68 potently inhibited PRMT5 $\left(\mathrm{IC}_{50}=517 \mathrm{nM}\right)$. (I) Western blot analysis of BCR-ABL and PRMT5 and its epigenetic marks in K562 cells treated with increasing concentrations of PJ-68. (J) PJ-68 inhibited $B C R-A B L$ gene transcription as measured by qRT-PCR analysis. (K and L) PRMT5 inhibition reduced mRNA and protein levels of BCR-ABL in CML CD34+CD38- cells and CD34+CD38+ cells $(n=3)$. (M) qRT-PCR analysis of Mir203 level in K562 cells treated with PJ-68 for 24 hours. (N and $\mathbf{0}$ ) PRMT5 inhibition by PJ-68 (N) or PRMT5 knockdown by shRNA (0) in K562 cells led to loss of recruitment of PRMT5 and its epigenetic marks to the promoter of Mir203 as determined by ChIP assays. Two-tailed Student's $t$ test was used for K, M, and N; 1-way ANOVA, post hoc intergroup comparisons, Tukey's test were performed for $\mathbf{C}, \mathbf{D}, \mathbf{F}$, and $\mathbf{~ J .}{ }^{*} P<0.05,{ }^{* *} P<0.01,{ }^{* *} P<0.0001$.

observed that forced overexpression of $B C R-A B L$ increased the endogenous PRMT5 protein level (Figure 1F). Intriguingly, transfection with pSG5-BCR-ABL (p190) but not HA-tagged BCR or pSG5-ABL upregulated endogenous PRMT5 at both the protein (Figure 1F) and mRNA levels (Supplemental Figure 1B). Thus, it is the $B C R-A B L$ fusion genes, but not $\mathrm{BCR}$ or $\mathrm{ABL}$, that upregulate PRMT5 expression.

We next examined whether depletion of BCR-ABL decreased PRMT5 expression. When IM-sensitive CML KBM5 and K562 cells were exposed to escalating concentrations of IM, PRMT5 protein levels were decreased in an IM concentration-dependent manner (Figure 1, G and $\mathrm{H}$, left). In contrast, PRMT5 protein levels were not altered by treatment of IM-resistant KBM5-T315I and uveal melanoma Mel270 cells (a control cell line not harboring $B C R-A B L)$ with IM at concentrations that abrogated BCRABL tyrosine kinase activity (Figure $1, \mathrm{G}$ and $\mathrm{H}$, right). Further, the mRNA levels of PRMT5 were decreased in $\mathrm{CD} 34^{+} \mathrm{CD} 38^{-}$and $\mathrm{CD} 34^{+} \mathrm{CD} 38^{+}$cell populations after treatment with IM (Figure 1I). Thus, pharmacological inhibition of BCR-ABL activity suppresses PRMT5 expression in CML cells.

Because endogenous PRMT5 proteins are distributed in both the cytoplasmic and nuclear compartments, we further examined the effect of IM on the subcellular distribution of PRMT5 in CML cells. We found that the level of PRMT5 was decreased in both the cytosolic and nuclear fractions following IM treatment (Supplemental Figure 1C). Immunofluorescence microscopy confirmed the decreased expression of PRMT5 in both the cytosolic and nuclear compartments (Supplemental Figure 1D) in IM-treated cells.

To further confirm the dependence of PRMT5 expression on BCR-ABL, we silenced BCR-ABL expression in K562 and in primary CD $34^{+}$cells from CML patients by transduction of lentiviral shRNA against $B C R-A B L$. The results showed that silencing $B C R$ $A B L$ depleted PRMT5 in K562 cells and in human CML CD34 $4^{+}$ cells (Figure 1, J and K). Therefore, BCR-ABL positively regulated PRMT5 expression in these cells.

PRMT5 expression might be regulated at various levels, such as protein phosphorylation, mRNA stability, and transcription $(30,31)$. We found that blocking BCR-ABL kinase activity by IM had minimal effect on both the protein and mRNA stability of PRMT5 (data not shown) but significantly decreased PRMT5 mRNA levels in K562 cells (Figure 1L). In contrast, forced overexpression of $B C R-A B L$ increased PRMT5 mRNA levels (Supplemental Figure 1B).
BCR-ABL-mediated PRMT5 transcriptional expression is STAT5 dependent. Given that the transcription factor STAT5, a key BCR$A B L$ downstream signaling molecule, is essential for $B C R-A B L$ transformation (32), we searched for and discovered 2 putative conserved STAT5 binding sites in the PRMT5 gene promoter. Using ChIP assays, we found that STAT5A and STAT5B were recruited to the promoter of PRMT5 (Figure 1M). Furthermore, treating K562 cells with IM abrogated the recruitment of STAT5A and STAT5B (Figure 1M). To further define the role of STAT5 in PRMT5 gene transcription, we knocked down STAT5 using shRNA against human STAT5A and STAT5B in K562 cells and then evaluated the expression of PRMT5. Knockdown of STAT5A and STAT5B expression greatly decreased the protein and mRNA levels of PRMT5 (Figure 1N and data not shown). These data suggest that BCR-ABL mediates PRMT5 transcriptional expression in a STAT5-dependent manner.

$B C R-A B L$ expression depends on PRMT5 in CML cells. To investigate the functional role of PRMT5 in CML, we transfected K562 cells with PRMT5 construct and collected single clones stably overexpressing PRMT5 (\#16 and \#19). The BCR-ABL protein level was greatly increased in the PRMT5-overexpressing cells (Figure 2A), suggesting that PRMT5 promotes BCR-ABL expression. We also determined whether PRMT5 worked in the setting of T315I BCR-ABL. Ectopic overexpression of PRMT5 in KBM5 cells harboring T315I BCR-ABL elicited an increased level of endogenous T315I BCR-ABL (Supplemental Figure 2, A and B).

Because the conserved arginine residue (R368) is essential for the methyltransferase activity of PRMT5, a catalytically dead PRMT5 was created by mutating the amino acid arginine 368 to alanine (R368A) (24). K562 cells were electrotransfected with pCMV-2B-Flag-PRMT5 or mutant pCMV-2B-Flag-PRMT5 (R368A). PRMT5 upregulated both protein and mRNA levels of BCR-ABL, which was dependent on intact methyltransferase activity of PRMT5 (Figure 2, B and C). To further explore the mechanism of $B C R-A B L$ gene transcription by PRMT5, we transiently cotransfected $293 \mathrm{~T}$ cells with the $B C R-A B L$ promoter reporter construct and the pCMV-2B-Flag-PRMT5 or mutant pCMV-2B-Flag-PRMT5 (R368A) for 24 hours. PRMT5 increased $B C R-A B L$ promoter reporter activity, which was also dependent on its methyltransferase activity (Figure 2D). Next, we examined the effect of PRMT5 knockdown on BCR-ABL expression. K562 cells were transduced with pLKO.1-GFP-shRNA or pLKO.1-GFPshPRMT5 lentivirus (Scramble, shPRMT5 \#1 and shPRMT5 \#2, respectively). Both the protein and mRNA levels of BCR-ABL were 

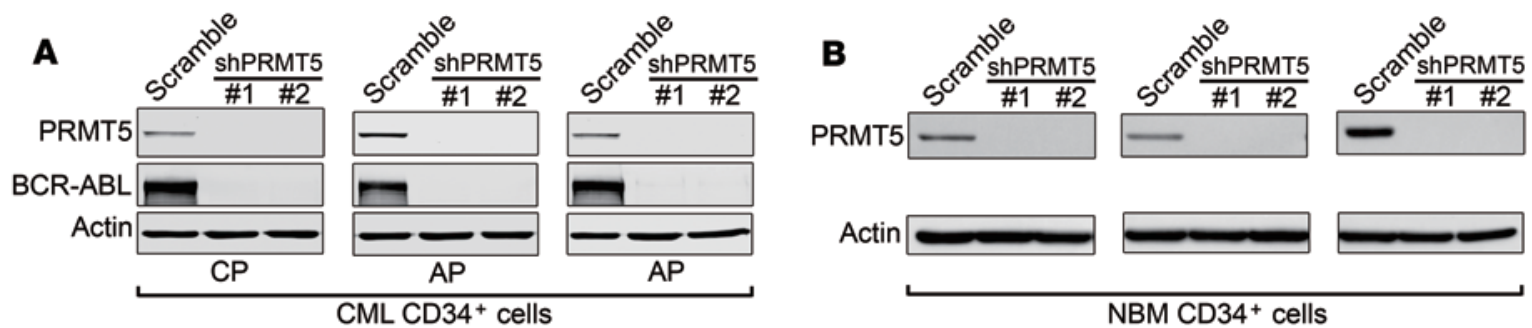

C

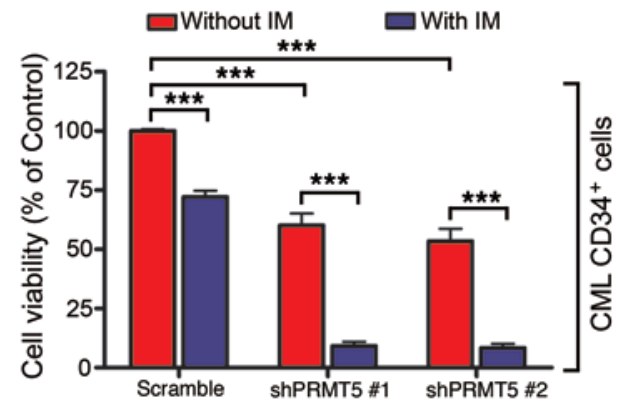

$\mathbf{E}$

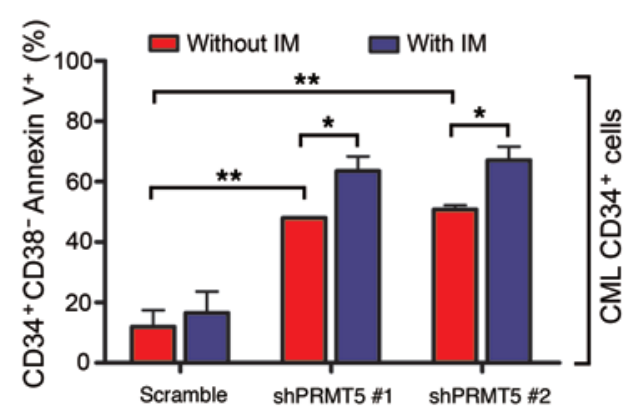

G

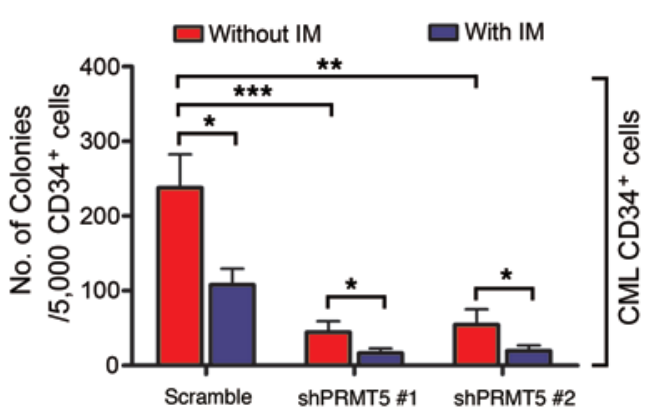

I

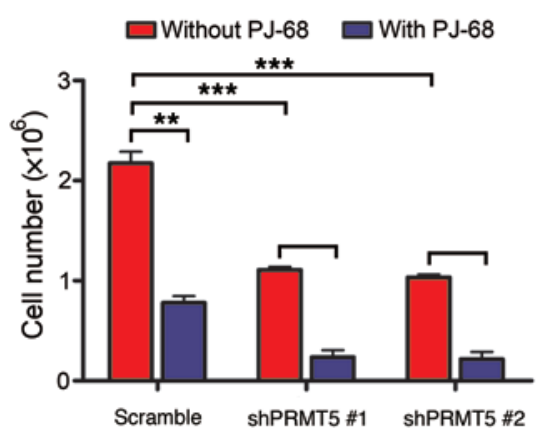

D

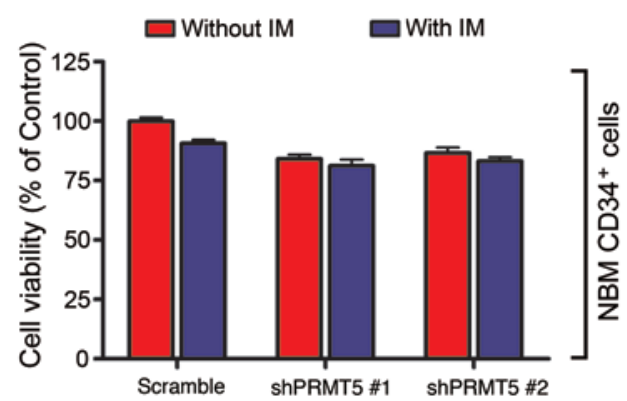

$\mathbf{F}$

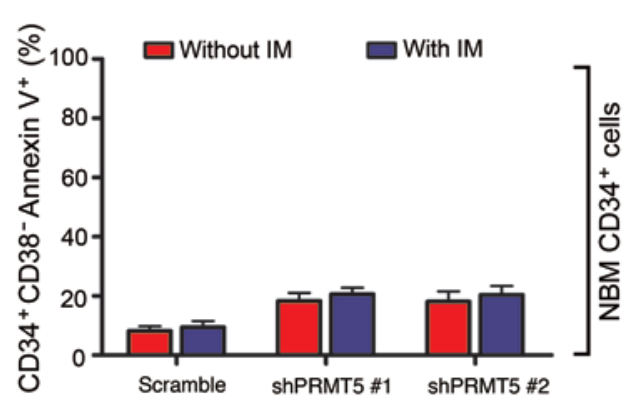

H
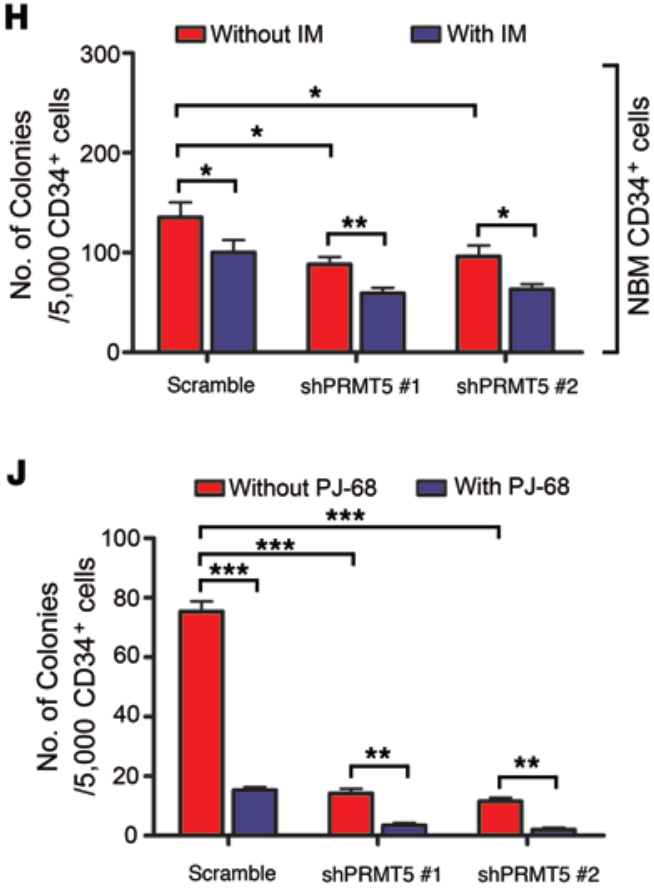
Figure 3. PRMT5 knockdown by lentiviral shRNA reduces growth, survival, and colony formation in human CML CD34+ cells. Human CML CD34+ (A, C, $\mathbf{E}$, and $\mathbf{G})$ versus NBM CD34 $(n=3$ each) (B, D, F, and $\mathbf{H}$ ) cells were transduced with control shRNA (Scramble), shPRMT5 \#1, or shPRMT5 \#2 for 48 hours, then treated with IM $(2.5 \mu \mathrm{M})$ for 24 hours. (A and B) Western blot analysis of PRMT5 and BCR-ABL. (C and $\mathbf{D})$ Cell viability was determined by MTS assay (Promega). (E and F) Apoptosis was detected by flow cytometry after dually staining CML and NBM CD34+ cells with annexin V-FITC and anti-CD38-PE. (G and $\mathbf{H})$ The same number of Scramble, shPRMT5 \#1, or shPRMT5 \#2 in CML and NBM CD34+ cells (5,000 cells/well) were seeded in methylcellulose medium (H4434). Colonies were counted at 14 days. (I and J) Forty-eight hours after 2 rounds of transduction with control shRNA (Scramble), shPRMT5 \#1, or shPRMT5 \#2 lentivirus, the human CML CD34+ cells $(n=3)$ were exposed to PJ-68 at $25 \mu \mathrm{M}$ for another 24 hours, and cell numbers (I) and CFC formation (J) were determined. ${ }^{*} P<0.05,{ }^{* *} P<0.01,{ }^{* *} P<0.0001,1$-way ANOVA, post-hoc intergroup comparisons, Tukey's test.

decreased in the PRMT5 stable knockdown cells (Figure 2, E and F). Similarly, PRMT5 knockdown by shRNA in KBM5-T315I cells decreased both the protein and mRNA levels of BCR-ABL (Supplemental Figure 2, C and D).

To conveniently perform in vitro and in vivo experiments involving the interference of PRMT5 function, we screened and identified a potent small molecule inhibitor of PRMT5 designated PJ-68 (chemical structure shown in Figure 2G). To gain an understanding of binding of PJ-68 to PRMT5, we performed molecular docking of PJ-68 to PRMT5 (Supplemental Figure 2E). The total score was 9.0382 (crash -1.6543). It has been shown that Glu499 and Glu508 located on a hairpin loop connecting $\beta 4$ and $\alpha \mathrm{F}$ (called "double-E" loop) are required for the enzymatic activity of PRMT5 (33). In addition, Phe379 might be important for PRMT's catalytic activity (33). These residues in the binding pocket were at $3 \AA$ distance from PJ-68, illustrating the electronic/hydrophobic/aromatic stacking interactions with the compound. Using an in vitro methyltransferase activity assay, we showed that PJ-68 inhibited the methyltransferase activity of PRMT5 with an $\mathrm{IC}_{50}$ of $517 \mathrm{nM}$ (Figure 2H). PJ-68 possessed no inhibitory activity against type I (PRMT1, 3, 4, 6, and 8) PRMT family members, indicating its specificity toward PRMT5 (Figure 2H). Similarly, PJ-68-treated K562 cells showed a concentration-dependent decrease in PRMT5 methyltransferase activity, as reflected by symmetrical arginine dimethylation of histone $\mathrm{H} 2 \mathrm{~A}$ and $\mathrm{H} 4$ and the absence of asymmetrical arginine dimethylation (H4R3ADM), which is catalyzed by type I enzyme PRMT1 (Figure 2I), further supporting the specificity of this inhibitor. In parallel experiments, PJ-68 treatment markedly decreased $B C R-A B L$ mRNA levels (Figure 2J). In addition, the level of BCR-ABL was significantly reduced in both $\mathrm{CD} 34^{+} \mathrm{CD} 38^{-}$cells and $\mathrm{CD} 34^{+} \mathrm{CD} 38^{+}$cells treated with PJ-68 (Figure 2, $\mathrm{K}$ and L).

Given that epigenetic silencing of Mir203 enhances the expression of the $B C R-A B L 1$ oncogene, and that PRMT5 inhibition leads to transcriptional derepression of miRNA $(34,35)$, we tested whether PRMT5 regulated BCR-ABL expression through miR-203 in CML cells. As shown in Figure 2M, PRMT5 inhibition upregulated miR-203 expression in K562 cells. ChIP experiments revealed an enrichment of PRMT5 and its epigenetic marks H4R3SDM and H3R8SDM at the Mir203 promoter, which was attenuated by treatment with PJ-68 or PRMT5 shRNA (Figure 2, N and O).

PRMT5 knockdown reduces growth, survival, and colony formation of CML CD34 ${ }^{+}$cells. To define the role of PRMT5 in CML stem/ progenitor cells, we transduced MACS bead-purified human CML or NBM CD $34^{+}$cells with a control shRNA (Scramble) or PRMT5 shRNA lentivirus for 48 hours, then treated them with IM for 24 hours. The results showed that the PRMT5 protein level was reduced in CML specimens, including cells from $2 \mathrm{AP}-\mathrm{CML}$ and $1 \mathrm{CP}-\mathrm{CML}$ patient (Figure 3A) and NBM CD34+ ${ }^{+}$cells (Figure 3B).
PRMT5 knockdown alone or in combination with IM significantly inhibited the cell viability of CML but not of NBM CD34 ${ }^{+}$cells (Figure 3, C and D). PRMT5 knockdown also significantly induced apoptosis in CML CD34 ${ }^{+} \mathrm{CD} 38^{-}$cells (Figure $3 \mathrm{E}$ ) but not in NBM CD $34^{+} \mathrm{CD} 38^{-}$cells (Figure 3F). This effect of PRMT5 knockdown on CML CD34 ${ }^{+}$cells was augmented with combined IM treatment. PRMT5 knockdown alone reduced CML colony-forming cells (CFCs) in the methylcellulose medium, which was further potentiated with IM combination (Figure 3G). In contrast, PRMT5 knockdown inhibited NBM CFC growth to a lesser extent (Figure $3 \mathrm{H})$. Thus, PRMT5 knockdown reduced CML CD $34^{+}$cell survival and growth, and these effects were augmented with IM combination. Additionally, combination treatments of the human CML CD34 ${ }^{+}$cells with PRMT5 shRNA and PJ-68 provoked a further reduction in cell growth colony-forming abilities (Figure 3, I and J).

PRMT5 knockdown inhibits in vivo growth of LSCs and prolongs survival of CML mice. In order to define the in vivo role of PRMT5 in CML LSCs, we used a mouse CML model with BM transduction and transplantation (36). C57BL/6 donor BM cells primed by 5 -fluorouracil (5-FU) were transduced with retroviral MSCVIRES-EGFP carrying p210 BCR-ABL. The transduced cells were transplanted into sublethally irradiated recipient mice, which developed CML in $\sim 2$ weeks. LSCs in BM and spleen of such CML mice would produce leukemia if they were intravenously transplanted into the secondary irradiated recipient mice (Figure 4A).

We knocked down PRMT5 by lentiviral shRNA in splenic $\mathrm{GFP}^{+}$ cells collected from the first generation of CML mice (Figure 4B) and then transplanted the modified splenic cells into sublethally irradiated (550 cGy) C57BL/6 recipient mice. The mice then received IM or vehicle for 2 weeks. PRMT5 knockdown alone or with IM significantly inhibited the splenomegaly of CML mice (Figure 4C). PRMT5 knockdown alone or with IM significantly prolonged the survival of CML mice (Figure $4 \mathrm{D}$ ). The populations of $\mathrm{GFP}^{+}$(BCR-ABL-expressing leukemia cells) and myeloid cells $\left(\mathrm{Gr}-1^{+} \mathrm{Mac}-1^{+}\right)$in $\mathrm{BM}$ were significantly reduced in the PRMT5-knockdown group and further reduced with IM treatment (Supplemental Figure 3, A and B). Flow cytometry analysis revealed that PRMT5 knockdown alone or with IM significantly decreased LSK ( Lin $^{-} \mathrm{Sca}-{ }^{+}{ }^{+} \mathrm{c}-\mathrm{Kit}^{+}$) cells, LT-HSCs (LSK Flt3 ${ }^{-} \mathrm{CD} 150^{+} \mathrm{CD} 48^{-}$), and ST-HSCs (LSK Flt3-CD150-CD48) in BM of CML mice (Figure $4, \mathrm{E}-\mathrm{H})$. The percentages of granulocyte-macrophage progenitors (GMPs, Lin'Sca- $1^{-} \mathrm{c}-\mathrm{Kit}^{+} \mathrm{CD} 34^{+} \mathrm{Fc} \gamma \mathrm{RII} / \mathrm{III}^{\mathrm{hi}}$ ) and common myeloid progenitors (CMPs, $\mathrm{Lin}^{-} \mathrm{Sca}-1^{-} \mathrm{c}-\mathrm{Kit}^{+} \mathrm{CD} 34^{+} \mathrm{F} \gamma \gamma \mathrm{RII} / \mathrm{IIII}^{\mathrm{lo}}$ ) were dramatically decreased in BM of CML mice (Supplemental Figure 3, C-E). Similar results were obtained in the splenic cells of CML mice (Supplemental Figure 3, F-L).

In a separate set of experiments, we examined the in vivo effect of PRMT5 knockdown in the LSK cell populations of CML mice. 
A
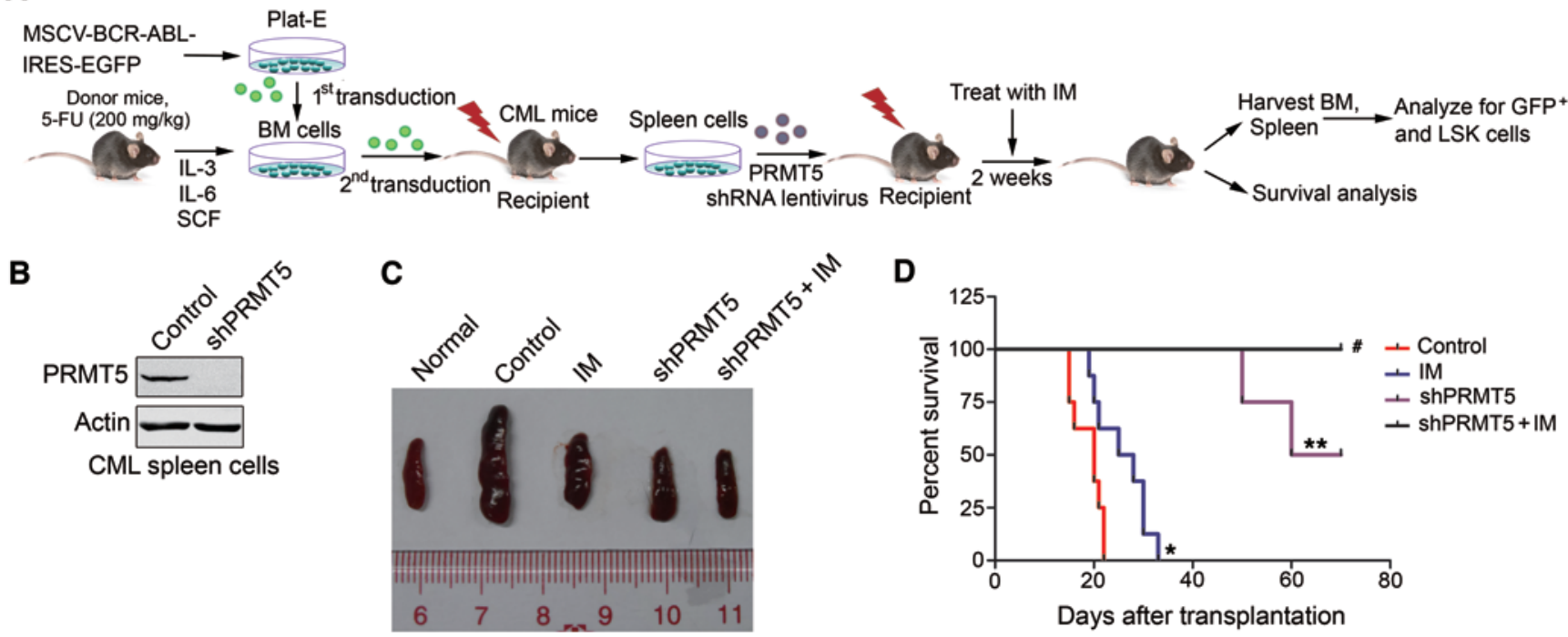

E

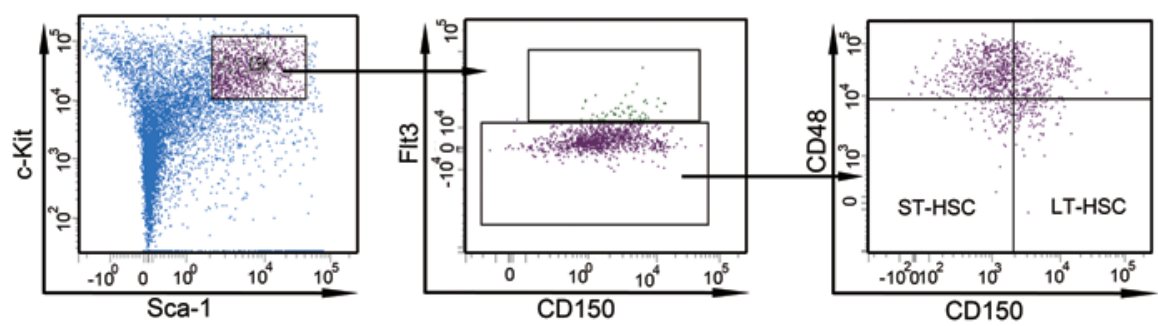

G

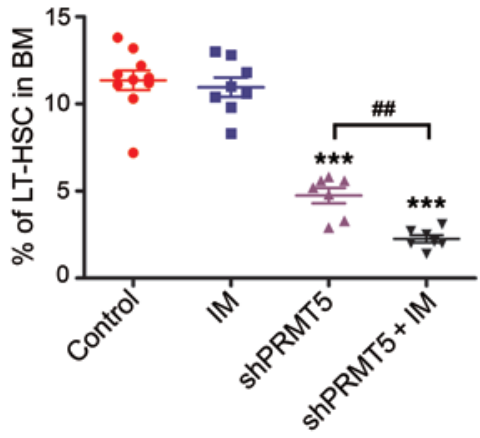

H

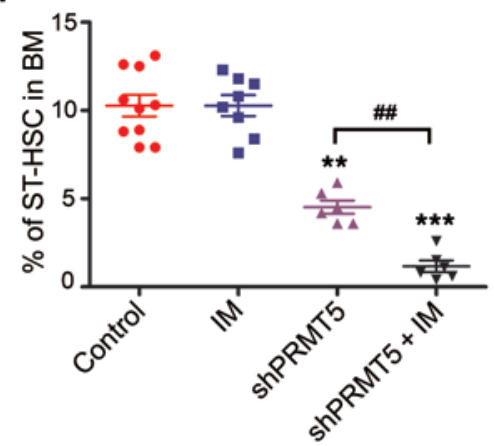

$\mathbf{F}$

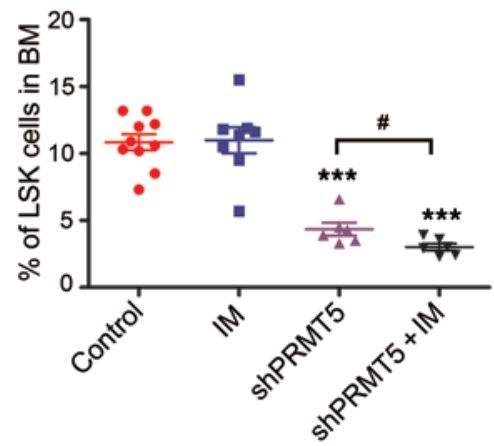

I

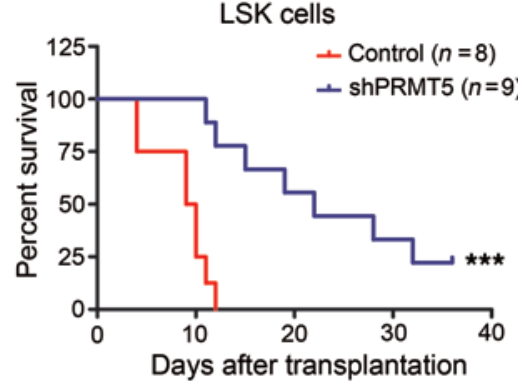

Figure 4. PRMT5 knockdown inhibits LSC growth in the CML mouse model. (A) Schematic strategy of evaluation of the in vivo effect of $P R M T 5$ knockdown on LSCs in the CML mouse model. (B) The effect of PRMT5 knockdown in spleen nucleated cells from CML mice was confirmed by Western blot analysis. (C) A representative photograph of spleens from each group. (D) Kaplan-Meier survival curves ( $n=8$ per group). ${ }^{*} P=0.0142$ (Control versus IM), ${ }^{* *} P=0.0029$ (Control versus shPRMT5), ${ }^{\#} P=0.0259$ (Control versus shPRMT5+IM), log-rank test. (E-H) Analysis of LSKs in BM cells of CML mice that received treatment with shPRMT5 \pm IM. Control, $n=10$; IM, $n=8$; shPRMT5, $n=6$; shPRMT5+IM, $n=6$. (E) Schema for analysis of LSK cells, LT-HSCs, and ST-HSCs. Results for the GFP+ population in BM: LSK cells (F), LT-HSCs (G), and ST-HSCs $(\mathbf{H}) .{ }^{*} P<0.01,{ }^{* * *} P<0.0001$, compared with control; ${ }^{*} P<0.05$, $\# \# P<0.01$, shPRMT5 compared with shPRMT5+IM, 1-way ANOVA, post hoc intergroup comparisons, Tukey's test. (I) LSK (Lin-Sca-1+c-Kit ${ }^{+}$) cells sorted by flow cytometry from the BM and spleens of first-generation CML mice underwent 2 rounds of transduction with lentiviral Scramble shRNA (Control) or shPRMT5 for 48 hours, and were then transplanted via tail veins into the secondary recipient mice, and survival curve was analyzed. Control, $n=8$; shPRMT5, $n=9 ;{ }^{* *} P=0.0001$, log-rank test.

We sorted the murine LSCs (LSK) from the nucleated cells of BM and spleen of the primary-generation CML mice by flow cytometry, and then transduced such LSK cells with lentiviral shRNA against murine PRMT5 for 48 hours and subsequently transplant- ed them into the secondary irradiated recipient C57BL/6 mice. Our results showed that knockdown of PRMT5 in the sorted LSK cells significantly prolonged the survival of the secondary recipients. The median survival for control versus shPRMT5-transduc- 
ed cells was 9.5 versus 22 days ( $P=0.0001$, log-rank test) (Figure $4 \mathrm{I})$. These results together suggest that silencing PRMT5 inhibits in vivo growth of CML LSCs and sensitizes them to IM treatment.

Pharmacological inhibition of PRMT5 reduces survival and self-renewal capacity of CML CD34+ cells. Next, we determined the effect of PJ-68 on survival of CML CD34 $4^{+}$cells and normal

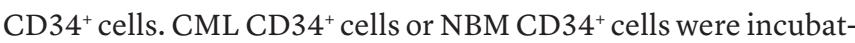
ed with PJ-68 $(25.0 \mu \mathrm{M})$ in the presence or absence of IM $(2.5 \mu \mathrm{M})$ for 24 hours. PRMT5 methyltransferase activity was decreased

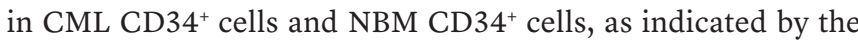
H2AR3SDM level after PJ-68 treatment alone or with IM (Figure 5A and Supplemental Figure 4A). In parallel, flow cytometry analysis of apoptosis based on dual staining with annexin $\mathrm{V}$ FITC and anti-CD38-phycoerythrin (anti-CD38-PE) antibodies revealed that $\mathrm{PJ}-68$ treatment increased the apoptosis of $\mathrm{CML}$ $\mathrm{CD} 4^{+}$cells (Figure 5, B and C) but had minimal effect on NBM $\mathrm{CD} 4^{+}$cells (Supplemental Figure 4, B and C). To further demonstrate the effectiveness of pharmacological inhibition of PRMT5 in quiescent CML LSCs, we labeled CML CD34 ${ }^{+} \mathrm{CD} 38^{-}$cells or $\mathrm{NBM} \mathrm{CD} 4^{+}$cells with CFSE in the presence of PJ-68 $(25.0 \mu \mathrm{M})$ for 96 hours. With PJ-68 treatment, apoptosis was significantly increased in quiescent CML LSCs (Figure 5D) versus in NBM $\mathrm{CD} 4^{+}$cells (Supplemental Figure 4D).

Given that self-renewal capacity is an important trait of LSCs, we determined whether inhibition of PRMT5 by PJ-68 impaired the self-renewal ability of CML stem cells by CFC/replating and long-term culture-initiating cell (LTC-IC) assay. Human CML $\mathrm{CD}^{+} 4^{+}$cells and NBM CD34 ${ }^{+}$cells were treated with PJ-68 for 24 hours and subjected to 14 -day serially plating CFC culture in drugfree methylcellulose medium. With PJ-68 treatment, CFC formation and replating ability was decreased more in CML than in NBM CD $34^{+}$cells (Figure 5E and Supplemental Figure 4E). Similarly, the decrease in the number of LTC-IC-derived colony-forming units was greater in CML BM cells (Figure 5F) than in NBM cells (Supplemental Figure 4F). Notably, combination of PJ-68 with IM significantly enhanced CML LTC-IC growth inhibition as compared with either agent alone (Figure $5 \mathrm{~F}$ ).

PRMT5 modulates the expression of cell cycle regulators in CML CD $34^{+}$cells. Western blot analysis showed that PRMT5 knockdown or pharmacological inhibition of PRMT5 activity with PJ-68 increased $\mathrm{p} 15^{\mathrm{INK} 4 \mathrm{~B}}$ and $\mathrm{p} 27^{\mathrm{KIP} 1}$ but not $\mathrm{p} 16^{\mathrm{INK} 4 \mathrm{~A}}$ and $\mathrm{p} 57^{\mathrm{KIP} 2}$ expression (Supplemental Figure 5, A and B). Similarly, PRMT5 knockdown or pharmacological inhibition of PRMT5 activity increased p15 ${ }^{I N K 4 B}$ mRNA levels in K562 cells (Supplemental Figure 5, C and D) and in CML CD34 ${ }^{+}$cells (Supplemental Figure 5, E and F). The $p 15^{I N K 4 B}$ gene is a negative regulator that prevents cell-cycle progression at the $G_{1} / S$ transition (37). Because PRMT5 specifically catalyzes H4R3SDM, which can recruit DNA methyltransferase DNMT3A to promoter regions of target genes to suppress gene expression (38), we determined whether PRMT5 mediated transcriptional repression of $\mathrm{p} 15^{\mathrm{INK} 4 \mathrm{~B}}$ by $\mathrm{H} 4 \mathrm{R} 3 \mathrm{SDM}$ and recruitment of DNMT3A at the $p 15^{I N K 4 B}$ promoter. ChIP assay showed enrichment of PRMT5 at the $p 15^{I N K 4 B}$ promoter, and PRMT5 knockdown by shRNA or inhibition by PJ-68 led to decreased enrichment of PRMT5 at the $p 15^{I N K 4 B}$ promoter (Supplemental Figure 5, G and H). Further, PRMT5 knockdown inhibited the enrichment of H4R3SDM (Supplemental Figure 5I) and DNMT3A (Supplemen- tal Figure 5J) at the promoter of $p 15^{I N K 4 B}$ in K562 cells. Consistent with this finding, the mRNA level of $p 15^{I N K 4 B}$ was dramatically increased following treatment with the DNMT3A inhibitor SGI1027 in K562 cells and CML CD34+ cells (Supplemental Figure $5, \mathrm{~K}$ and $\mathrm{L}$ ). These results suggest that PRMT5 may modulate the expression of cell cycle regulators including $p 15^{I N K 4 B}$ gene by affecting histone arginine methylation.

Pharmacological inhibition of PRMT5 reduces growth of CML LSCs and prolongs survival of CML mice. To recapitulate the effect of pharmacological inhibition of PRMT5 on CML in vivo, we used the aforementioned $\mathrm{BM}$ transduction/transplantation (BMT) CML mouse model (36). The CML mice were then treated with PJ-68 (25 mg/kg/d and $50 \mathrm{mg} / \mathrm{kg} / \mathrm{d}$, i.p.), IM $(100 \mathrm{mg} / \mathrm{kg} / \mathrm{d}$, p.o.), or both (Figure 6A). Vehicle-treated control mice died of CML-like disease approximately 2 weeks after BMT, manifesting splenomegaly (Supplemental Figure 6A), elevated leukocyte counts predominated by myeloid leukemia $\left(\mathrm{GFP}^{+}\right)$cells, and lung hemorrhage. We next examined the bulk leukemia cells and stem/ progenitor cells in CML mice. Splenomegaly was not observed in the mice that received PJ-68 or both PJ-68 and IM (Supplemental Figure 6A). PJ-68- or IM-treated CML mice showed significantly prolonged survival, and PJ-68 plus IM further extended survival as compared with each agent alone (Figure 6B). The proportion of BCR-ABL-expressing $\left(\mathrm{GFP}^{+}\right)$leukemia cells and myeloid cells $\left(\mathrm{Gr}-1^{+} \mathrm{Mac}-1^{+}\right)$in BM cells was significantly reduced in the PJ-68treated CML mice; combining PJ-68 with IM further reduced these cell populations (Figure 6, C and D).

PJ-68 alone or in combination with IM, but not IM alone, markedly decreased the proportion of LSK cells, LT-HSCs, and ST-HSCs (Figure 6, E-G), as well as GMP and CMP in BM cells (Figure 6, H and I). Similar results were obtained in spleen cells (Supplemental Figure 6, B-J).

Pharmacokinetic (PK) study showed that PJ-68 $C_{\max }$ values were $3.57 \pm 0.08 \mu \mathrm{M}(1,264.4 \pm 28.4 \mathrm{ng} / \mathrm{ml})$ and $7.88 \pm 0.31 \mu \mathrm{M}$ $(2,788.5 \pm 109.7 \mathrm{ng} / \mathrm{ml})$, respectively, after a single oral or intravenous administration in SD rats (Supplemental Figure 6K and Supplemental Table 1). Such plasma concentrations of PJ-68 are postulated to be sufficient to block the histone methyltransferase activity of PRMT5 in vitro with an $\mathrm{IC}_{50}$ value of $517 \mathrm{nM}$ (Figure 2H).

Pharmacological inhibition of PRMT5 activity decreases the frequency of in vivo CML LSCs. To further analyze the frequency of CML LSCs subjected to treatments, we used a limiting dilution assay of BM cells in the secondary transplantation (Figure 7A). Treatment with PJ-68 alone or in combination with IM greatly decreased the engraftment of $\mathrm{GFP}^{+}$cells and the frequency of CML stem cells in the secondary recipients at 16 weeks after BMT (Figure 7, B and C, and Table 1).

PRMT5 inhibition reduces long-term multilineage engraftment of human CML CD34+ cells in NSI mice. We also determined the effect of ex vivo treatment with PJ-68 in human CML CD34 ${ }^{+}$cells on their ability to be engrafted in NOD-scid Il2 $\mathrm{rg}^{-/-}$(NSI) mice (Figure 8A). PJ-68 treatment reduced the engraftment of human $\mathrm{CML} \mathrm{CD} 45^{+}$cells in BM (Figure 8, B and C) and spleen (Figure $8 \mathrm{D})$ at 12 weeks after transplantation. The engrafted human CML CD $45^{+}$cells sorted by flow cytometry from NSI murine BM cells expressed BCR-ABL, and PJ-68 treatment markedly decreased $B C R-A B L$ mRNA level (Figure $8 \mathrm{E}$ ). The proportion of engrafted 
A

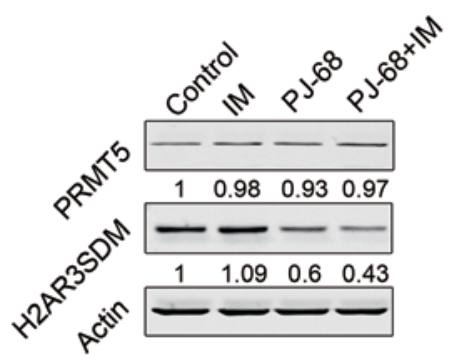

B

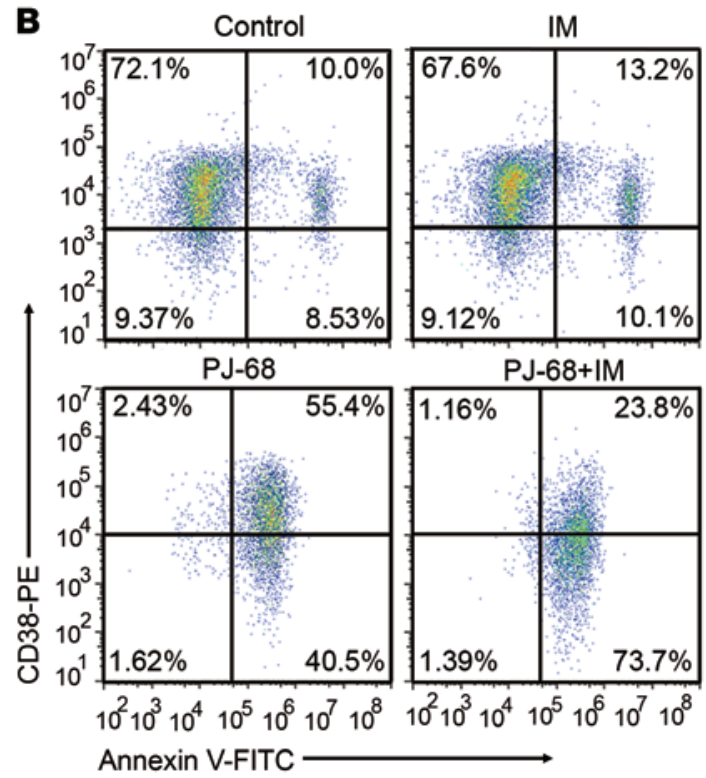

C

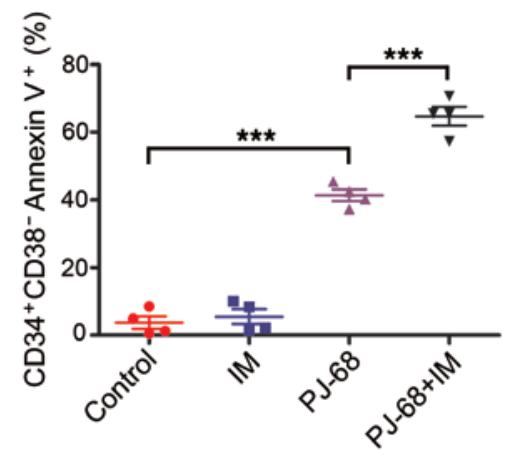

CML CD34+ cells

D
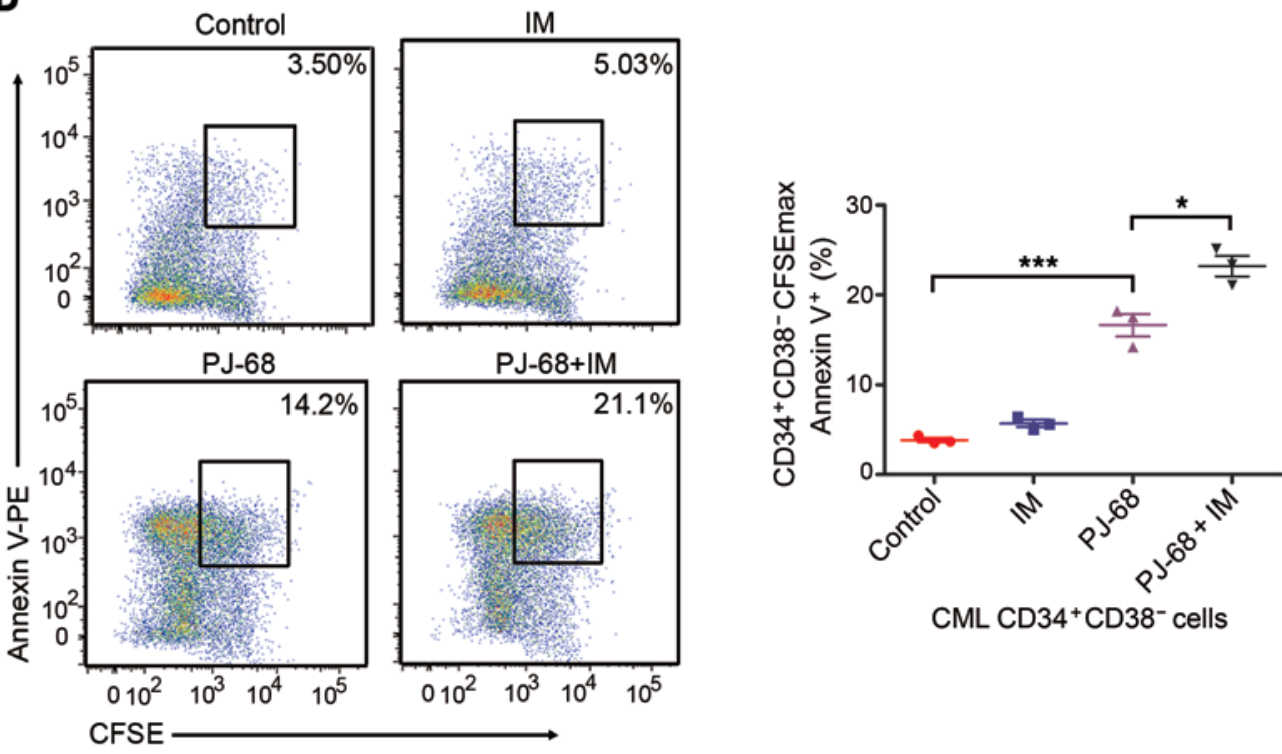

E

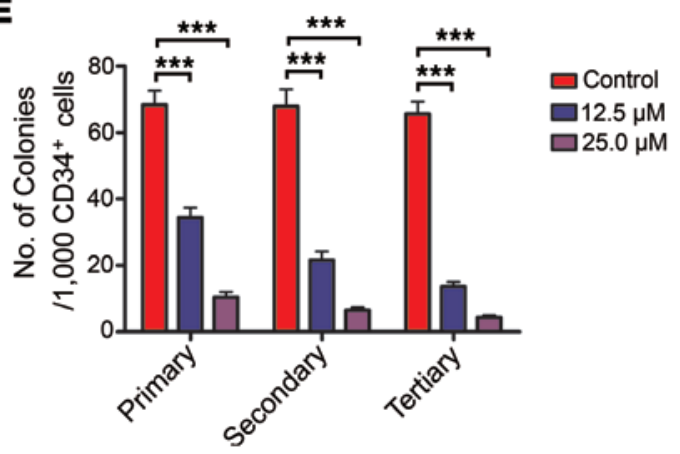

$\mathbf{F}$

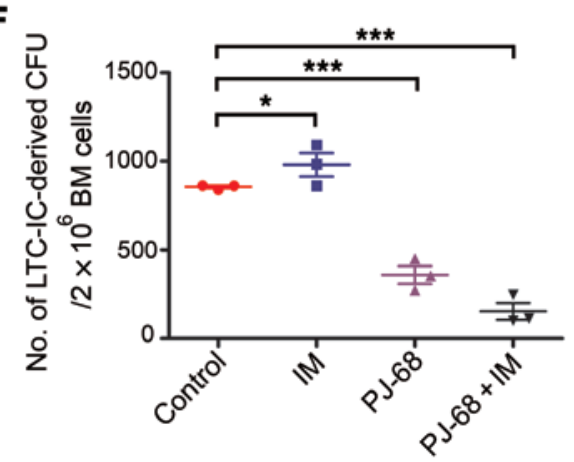


Figure 5. Pharmacological inhibition of PRMT5 activity induces apoptosis and suppresses self-renewal of human CML CD34 ${ }^{+}$cells. (A) Human CML CD34 ${ }^{+}$cells were treated with PJ-68, IM, or a combination for 24 hours, and Western blot analysis of protein levels of PRMT5 and its arginine methylation mark H2AR3SDM was performed. (B) Representative flow cytometry plots for apoptosis in human CML CD34+ cells. (C) CML CD34+ cells $(n=4)$ were treated with PJ-68 alone and in combination with IM (2.5 $\mu \mathrm{M})$ for 24 hours, and apoptosis was analyzed by flow cytometry after staining with annexin $\mathrm{V}$ FITC and anti-CD38-PE. (D) Sorted human CML CD34+CD38- cells $(n=3)$ were labeled with CFSE, then cultured with PJ-68 \pm IM for 96 hours, and apoptotic cells were analyzed by flow cytometry after labeling with annexin V-PE. (E) Pharmacological inhibition of PRMT5 lessened the serial replating capacity of CML LSCs. Human CML CD34+ cells $(n=3)$ were treated with 2 different concentrations of PJ-68 for 24 hours, washed with PBS, and counted; 1,000 cells were plated in methylcellulose medium. Colonies were counted on day 14. The cells were harvested and counted, 1,000 cells were replated for the second and third rounds, and colonies were counted on day 14 after each round. (F) Pharmacological inhibition of PRMT5 activity lessened the LTC-IC capacity of CML LSCs. $2 \times 10^{6}$ human CML cells $(n=3)$ were seeded in LTC-IC medium onto irradiated M2-10B4 cells and treated with PJ-68 (25.0 $\mu$ M) with or without IM $(2.5 \mu \mathrm{M})$ for 1 week, and cells were cultured for 5 more weeks in LTC-IC medium with weekly half drug-free medium changes. After 6 weeks, cells were harvested and plated into MethoCult H4435. LTC-IC-derived colonies were counted after 14 days. ${ }^{*} P<0.05$, ${ }^{* *} P<0.0001,1$-way ANOVA, post hoc intergroup comparisons, Tukey's test.

human CML CD33 $3^{+}$and $\mathrm{CD}_{14}{ }^{+}$myeloid cells in NSI murine BM cells was decreased following PJ-68 treatment (, F and G). Thus, PJ-68 treatment may selectively target human CML CD $34^{+}$cells with engraftment capacity in vivo.

PRMT5 activates Wnt/ $\beta$-catenin signaling in $C M L C D 34^{+}$cells. Canonical Wnt signaling is activated by binding of Wnt proteins to their respective cell surface receptors Frizzled and LRP6 on HSCs, which leads to activation and nuclear translocation of $\beta$-catenin, complex formation with TCF4, and transcription of target genes (39). $\beta$-Catenin is a critical protein controlling the self-renewal capacity of CML LSCs (13). We wondered whether PRMT5 inhibition blocked the Wnt/ $\beta$-catenin pathway in $\mathrm{CML} \mathrm{CD} 34^{+}$cells. We found that PRMT5 knockdown reduced the protein levels of $\beta$-catenin, dishevelled homolog 3 (DVL3, an upstream positive regulator of $\beta$-catenin), and target genes (e.g., cyclin D1, MYC, and LEF1) of $\beta$-catenin in K562 cells (Figure 9A). Similar results were obtained in PJ-68-treated K562 and primary human CML CD34 ${ }^{+}$ cells (Figure 9, B and C). In contrast, PRMT5 overexpression activated the Wnt/ $\beta$-catenin pathway in CML cells (Figure 9D). The intracellular activated $\beta$-catenin level in the PRMT5 knockdown or PJ-68-treated K562 cells was detected by fluorescence-tagged antibody followed by flow cytometry; loss of PRMT5 function significantly reduced intracellular activated $\beta$-catenin level (Figure 9E and Supplemental Figure 7A).

Nuclear $\beta$-catenin in complex with TCF4/LEF1 transcription factors can activate target genes whose promoters contain the regulatory elements (39). Therefore, we examined whether PJ-68 treatment reduced TCF4/LEF1-dependent transcription. We transduced primary human CML CD $34^{+}$cells with TOP- or FOP-lentivirus for 48 hours, and then the cells were treated with increasing concentrations of PJ-68 for 24 hours. Inhibition of PRMT5 by PJ-68 substantially decreased TOP luciferase activity but did not affect that of the FOP promoter, which has the mutated TCF4-binding sites (Figure 9F). Alternatively, K562 cells stably transduced with shRNA targeting PRMT5 were transfected with TOPflash and FOPflash constructs and pEFRenilla-luc for 24 hours. PRMT5 knockdown reduced TCF4/LEF1-dependent transcription (Supplemental Figure 7B). In contrast, ectopic expression of PRMT5 activated TCF4/LEF1-dependent transcriptional activity (Supplemental Figure 7C).

Next, we determined whether PRMT5 inhibition disrupted the interaction between $\beta$-catenin and TCF4. Coimmunoprecipitation (Co-IP) experiments showed that PRMT5 inhibition reduced $\beta$-catenin and TCF4 binding in the cells with PRMT5 stable knock- down (Figure 9G). Quantitative real-time PCR (qRT-PCR) analysis showed that PRMT5 knockdown reduced expression of the Wnt target genes AXIN2, MYC, LEF1, and CCND1 (Supplemental Figure 7D). Conversely, PRMT5 overexpression increased expression of these Wnt target genes (Supplemental Figure 7E).

Because the DVL3 but not DVL1 and DVL2 level was reduced by PRMT5 inhibition (Figure 9, A-C and data not shown), whereas overexpression of PRMT5 increased the DVL3 level (Figure 9D), we explored the mechanism by which PRMT5 positively affected DVL3 level. K562 cells were pretreated with the proteasome inhibitor MG132 $(1.0 \mu \mathrm{M})$ for 2 hours, followed by PJ-68 $(15.0 \mu \mathrm{M}$ or $20.0 \mu \mathrm{M})$ treatment for 24 hours. MG132 did not prevent PJ-68-mediated reduction of DVL3 protein (Supplemental Figure 7F). By contrast, PRMT5 activated DVL3 gene transcription, and PJ-68 treatment greatly inhibited the DVL3 mRNA level in K562 cells (Figure 9, $\mathrm{H}$ and I). Furthermore, we set out to examine whether the promoter of DVL3 was epigenetically regulated by PRMT5. Given that PRMT5 mediates symmetric dimethylation of histone H3R2 (H3R2SDM) and recruits WDR5 to promote H3K4 methylation and gene activation $(40,41)$, we determined the level of H3R2SDM and found that it was significantly decreased after PRMT5 knockdown or PJ-68 treatment in K562 cells (Figure 9J) or CML CD34+ cells (Supplemental Figure 7G). Further, ChIP experiments with antibodies specific for PRMT5, WDR5, H3R2SDM, and H3K4me3 revealed a significant enrichment of PRMT5 and its epigenetic marks at the DVL3 gene promoter, while knockdown of PRMT5 led to loss of recruitment of PRMT5 and its epigenetic marks on the DVL3 gene promoter in K562 cells (Figure 9K). These data indicate that the DVL3 gene promoter may be epigenetically regulated by PRMT5. Hence, PRMT5 may activate $\mathrm{Wnt} / \beta$-catenin signaling via transcriptional regulation of DVL3 in CML cells.

\section{Discussion}

LSCs are insensitive to IM and are seeds of resistance to TKI therapy and relapse in CML. Little is known about the key regulatory proteins and effective therapeutic targets for self-renewal of LSCs. Here, we discovered a reciprocal positive feedback regulation between PRMT5 and BCR-ABL. BCR-ABL activated PRMT5 transcriptional expression via STAT5 during malignant transformation in normal CD34 ${ }^{+}$cells. PRMT5 upregulated BCR-ABL expression through miR-203 in CML cells. PRMT5 was overexpressed in human CML CD34 ${ }^{+}$cells. Knockdown of PRMT5 by a specific shRNA or pharmacological inhibition of PRMT5 enzyme activity by PJ-68 treatment reduced survival, serially replating capacity, and LTC-IC in human 
A
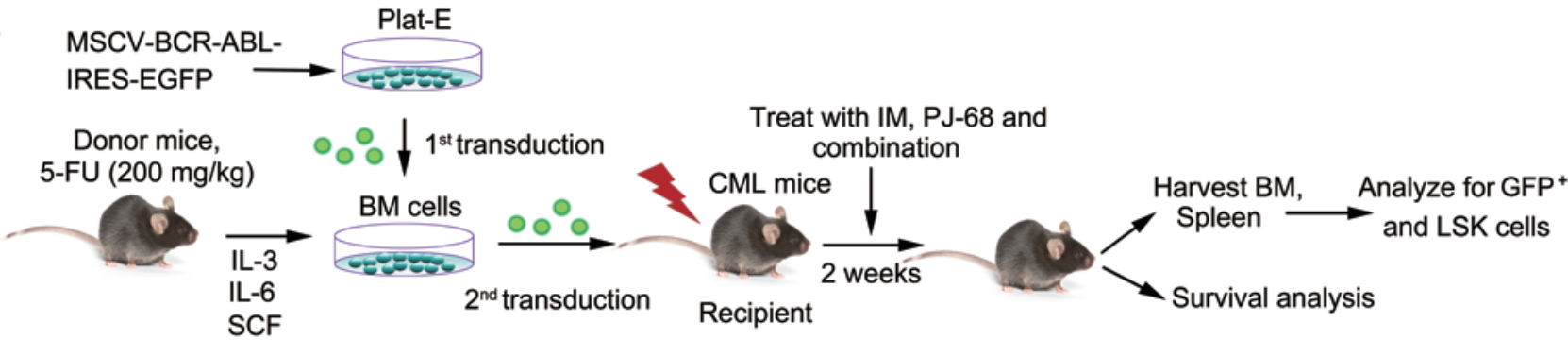

B

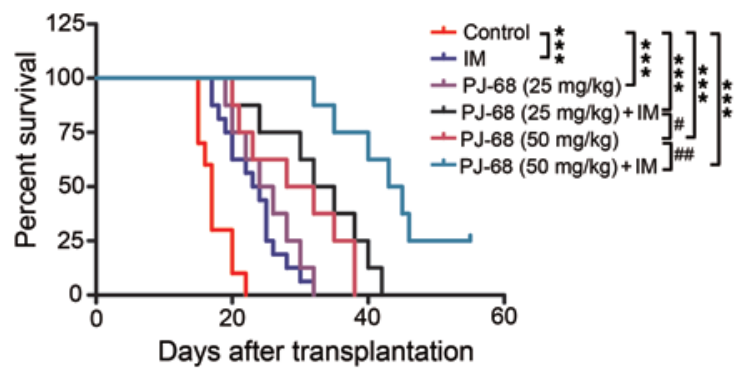

C

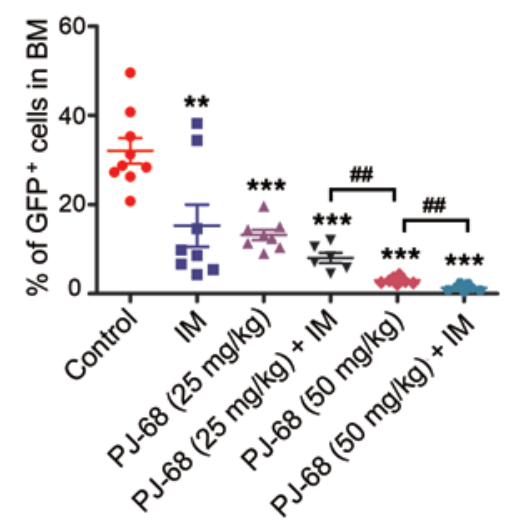

D

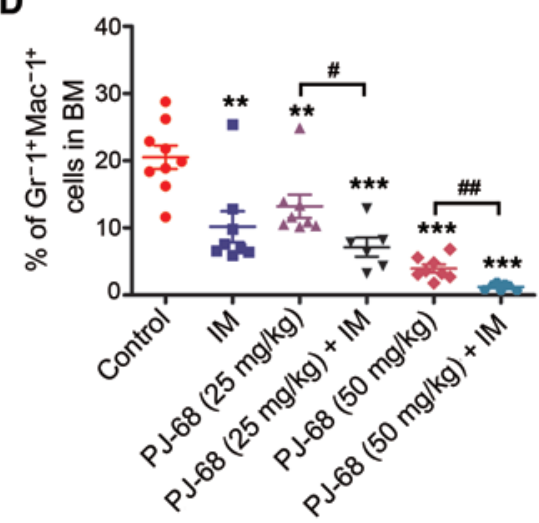

G

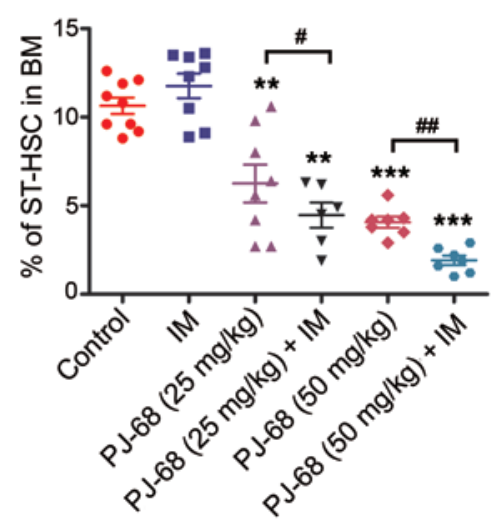

E

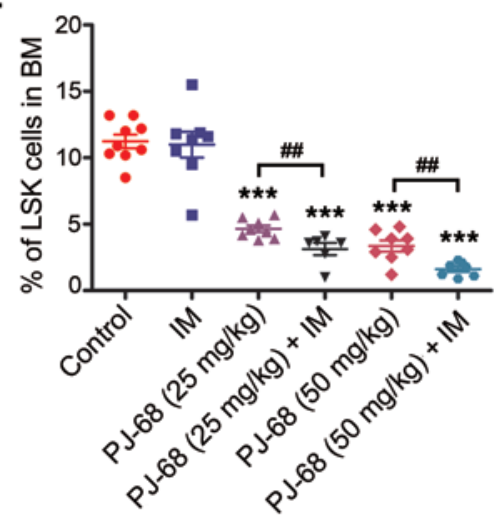

H

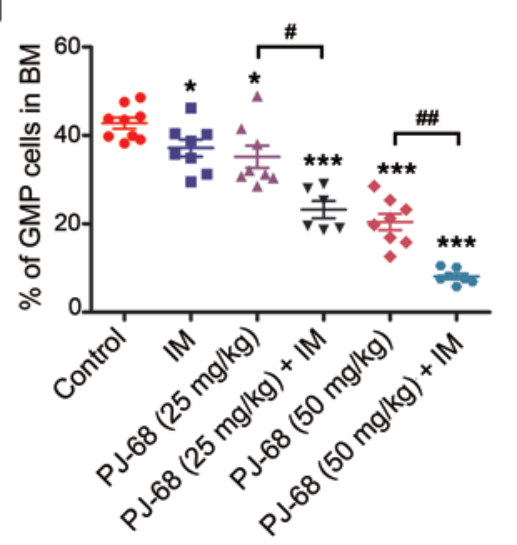

$\mathbf{F}$

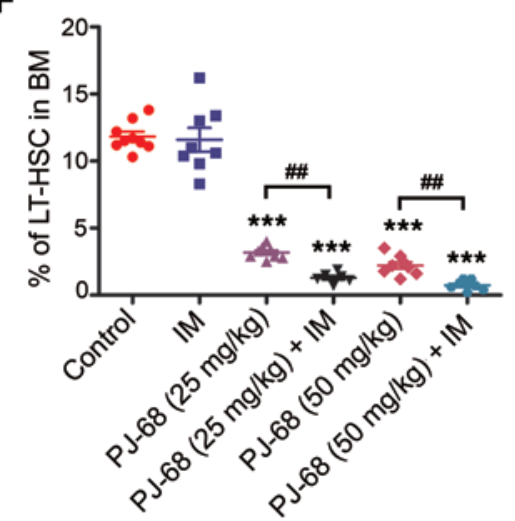

I

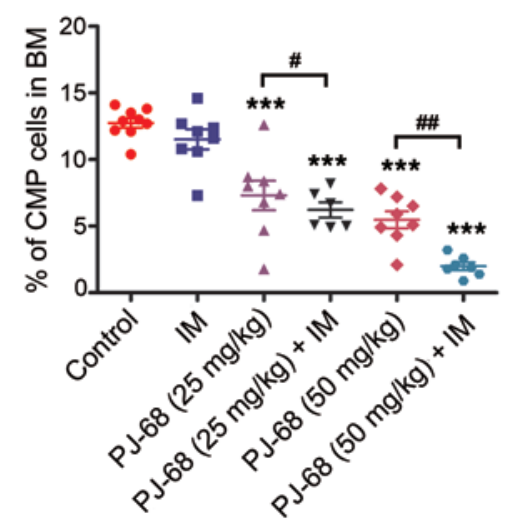

Figure 6. Pharmacological inhibition of PRMT5 activity reduces growth of CML stem cells in mice. (A) A schematic strategy for testing the efficacy of PJ-68 in combination with IM in a CML mouse model. (B) Kaplan-Meier survival curves of CML mice treated with IM, PJ-68, or their combination. Control $(n=10)$ versus IM $(n=16),{ }^{* * *} P=0.0003$; control versus PJ-68 $(25 \mathrm{mg} / \mathrm{kg})(n=8),{ }^{* * *} P=0.0010$; control versus PJ-68 $(25 \mathrm{mg} / \mathrm{kg})+\mathrm{IM}(n=8)$, ${ }^{* * *} P=0.0001$; control versus PJ-68 (50 mg/kg) $(n=8)$, ${ }^{* * *} P=0.0002$; control versus PJ-68 (50 mg/kg)+IM $(n=8)$, ${ }^{* * *} P<0.0001$, log-rank test. (C-C) Bulk leukemia cells and stem/progenitor cells in BM were analyzed by flow cytometry. Control $(n=9), \mathrm{IM}(n=8), \mathrm{PJ}-68(25 \mathrm{mg} / \mathrm{kg})(n=8), \mathrm{PJ}-68(25 \mathrm{mg} / \mathrm{kg})+\mathrm{IM}(n=6), \mathrm{PJ}-68$ $(50 \mathrm{mg} / \mathrm{kg})(n=8)$, PJ-68 (50 mg/kg)+IM ( $n=7)$. (C) GFP+ (leukemia) cells and (D) GFP+ myeloid (Gr-1+Mac-1+) cells in the BM. Results for the GFP+ population in the BM are shown: LSK cells (E), LT-HSCs (F), and ST-HSCs (G). (H) GMPs and (I) CMPs in the BM. ${ }^{*} P<0.05,{ }^{* *} P<0.01,{ }^{* * *} P<0.0001$, compared with control; ${ }^{P} P<0.05,{ }^{\#} P<0.01, P J-68$ compared with PJ-68+IM, 1-way ANOVA, post hoc intergroup comparisons, Tukey's test. 


\section{Table 1. Limiting dilution assay of mouse LT-HSCs after drug treatment}

Cell number

$\begin{array}{lcccc} & \text { Control } & \text { IM } & \text { PJ-68 } & \text { Combination } \\ 2 \times 10^{6} & 8 / 8 & 7 / 7 & 5 / 6 & 1 / 6 \\ 1 \times 10^{6} & 7 / 7 & 4 / 5 & 3 / 6 & 0 / 8 \\ 5 \times 10^{5} & 7 / 8 & 2 / 2 & 1 / 6 & 0 / 8 \\ \text { LT-HSC frequency } & 1 / 223,325 & 1 / 426,051 & 1 / 1,437,773 & 1 / 3,283,885\end{array}$

The fraction of mice represents the incidence of engraftment at 16 weeks after the secondary transplantation.

ABL to activate PRMT5 transcription, which may be plausible, because STAT5 is not only an important downstream target of BCR-ABL but also involved in the maintenance of CML LSC survival and IM resistance $(46,47)$. Similar to our findings, Yuan et al. reported that STAT5 was a mediator in BCR-ABL activating the gene transcription of SIRT1, an important epigenetic regulator of protein acetylation (48).

Therefore, our findings of a positive feedback loop between BCR-ABL and PRMT5 promote the understanding of the BCR-ABL regulatory network.

CML stem cells. Similarly, inhibition of PRMT5 prolonged the survival of CML mice and reduced the growth of CML LSCs in mice. In addition, pharmacological inhibition of PRMT5 reduced long-term engraftment of human CML CD34 ${ }^{+}$cells in NSI mice. Inhibition of PRMT5 abrogated Wnt/ $\beta$-catenin signaling in $\mathrm{CML} \mathrm{CD} 34^{+}$cells. Histone protein arginine methylation may be a novel regulation mechanism to control self-renewal of LSCs, and targeting PRMT5 helped effectively eradicate CML LSCs. Additionally, we identified a potent lead compound against PRMT5 activity and characterized its in vitro and in vivo activity against CML LSCs (Figure 10).

Positive feedback loop between BCR-ABL and PRMT5. A wide spectrum of substrates of BCR-ABL tyrosine kinase has been demonstrated $(42,43)$. The half-life of BCR-ABL transcripts seems to be a predictor of prognosis in patients with CML in response to TKIs (44). However, little is known about the mechanism regulating BCR-ABL expression. Our results revealed that PRMT5 is a positive regulator of $\mathrm{BCR}-\mathrm{ABL}$ expression in various settings, such as WT BCR-ABL, T315I mutation, and primary AP-CML CD34 ${ }^{+}$ cells. Conversely, knockdown of PRMT5 by shRNA or blocking its arginine methyltransferase activity by the small-molecule inhibitor PJ-68 greatly reduced $B C R-A B L$ gene transcription. Previous study has shown that miR-203, a potent negative regulator of $B C R-A B L$ oncogene transcription, is transcriptionally modulated by hypermethylation of the Mir203 promoter (35). Given that methylation on histone arginine residues governs the recruitment of histone protein on promoters of miRNAs, which subsequently determine switch-on or -off of their transcription $(24,45)$, it was postulated that PRMT5 might be recruited to regulate miR203 through histone arginine methylation. Indeed, our results showed that silencing PRMT5 by shRNA or pharmacological inhibition led to loss of enrichment of PRMT5 and its epigenetic marks H3R8SDM and H4R3SDM on the Mir2O3 promoter, and restoration of miR-203. These results suggest that histone protein methylation may represent another type of epigenetic means for expression of miR-203, which is a critical regulator of BCR-ABL. To our knowledge, this is the first report of a link between BCR$\mathrm{ABL}$ expression and a class of epigenetic methylation modifications on histone protein arginine residues.

Our data also suggest that BCR-ABL reciprocally regulates the expression of PRMT5. During the transformation process of $\mathrm{NBM} \mathrm{CD} 34^{+}$cells triggered by transduction of $B C R-A B L, P R M T 5$ expression was strikingly elevated. By contrast, silencing $B C R-A B L$ or inactivating BCR-ABL downregulated PRMT5 level. Further, our results support that STAT5 functions as a mediator for BCR-
PRMT5 modulates the expression of cell cycle regulators in CML CD $34^{+}$cells. PRMT5 knockdown remarkably increased expression of $\mathrm{p} 15^{\mathrm{INK} 4 \mathrm{~B}}$ and $\mathrm{p} 27^{\mathrm{KIP1} 1}$ (Supplemental Figure 5). p15 ${ }^{\mathrm{INK} 4 \mathrm{~B}}$ inhibits the self-renewal of LSCs in myeloid leukemia (49). Monitoring the evolution of xenografted human BCR-ABL lymphoblastic LSCs in SCID mice indicates that loss of CDKN2A/B is associated with increased LSC proportion and shortened survival (50). PRMT5 knockdown or pharmacological inhibition of PRMT5 activity increased $p 15^{I N K 4 B}$ mRNA levels, in association with a mechanism by which enrichment of H4R3SDM and DNMT3A is reduced at the promoter of $p 15^{I N K 4 B}$ in CML cells.

$\mathrm{p} 27^{\mathrm{KIP} 1}$ has been demonstrated to be an endogenous CDK inhibitor that negatively regulates the cell cycle and the self-renewal and survival of CML LSCs $(12,51)$. PRMT5 inhibition activated $\mathrm{p} 27^{\mathrm{KIP1}}$ expression, which may also contribute to inhibition of CML LSCs. Collectively, the increased p15 $5^{\mathrm{INK} 4 \mathrm{~B}}$ and $\mathrm{p} 27^{\mathrm{KIP} 1}$ expression by PRMT5 inhibition may facilitate eradication of LSCs in CML.

PRMT5 is an oncogenic protein in CML LSCs. The body of evidence we present here supports that PRMT5 is required for survival and self-renewal of CML LSCs, and that inhibition of PRMT5 eliminated the quiescent LSCs and impaired their self-renewal in human CML LSCs and in a CML mouse model. The effectiveness of targeting PRMT5 on LSCs may have particular implications for AP-CML patients, in which additional oncogenic drivers are assumed to be involved. The underlying mechanism may be associated with $\beta$-catenin, a central component of the Wnt/ $\beta$-catenin pathway that regulates stemness of CSCs in a wide range of malignancies (e.g., leukemia, breast cancer, and colorectal cancer) $(13,52,53)$.

Regarding the mechanism whereby PRMT5 elevates $\beta$-catenin levels, our data support that PRMT5 may execute this function in an indirect manner, i.e., via regulating the level of DVL3. Among the 3 isoforms of DVL protein, DVL1 was undetectable in K562 cells (data not shown), and DVL2 was not altered in PRMT5-silenced CML cells (Figure 9). However, PRMT5 inhibition markedly decreased DVL3 mRNA and protein levels (Figure 9). Our results suggest that increased PRMT5 in CML CD34 ${ }^{+}$cells may promote transcription of the DVL3 gene, which elicits increased stability of $\beta$-catenin in the absence of Wnt. Hence, decreased $\beta$-catenin and increased $\mathrm{p} 15^{\mathrm{INK} 4 \mathrm{~B}}$ levels by PRMT5 knockdown or inhibition may contribute to the elimination of CML LSCs.

PRMT5 inhibitors. Among the 11 members of the PRMT family, only PRMT5 has a solved crystal structure (33). We identified a potent PRMT5 small-molecule inhibitor, PJ-68, that was 
A
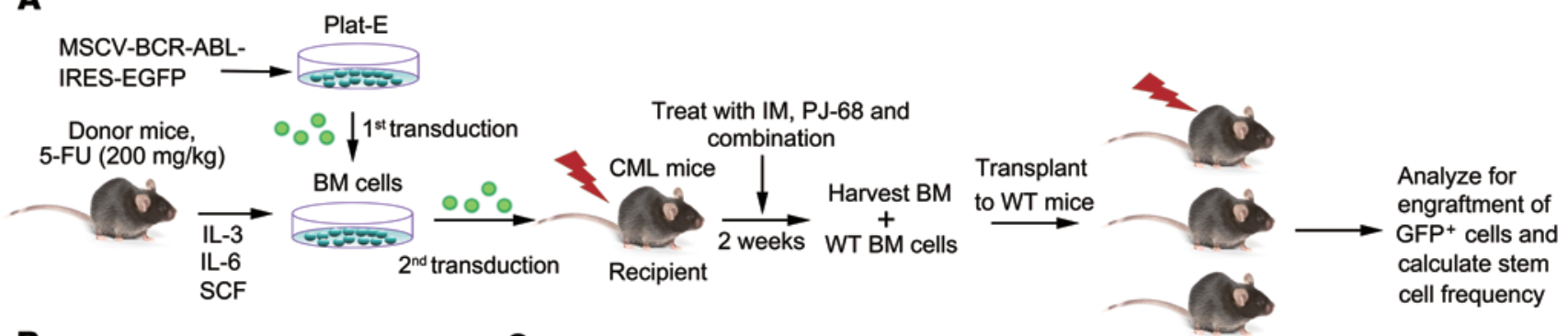

B

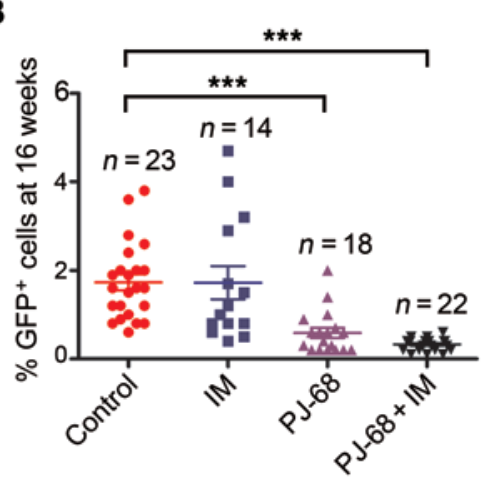

C

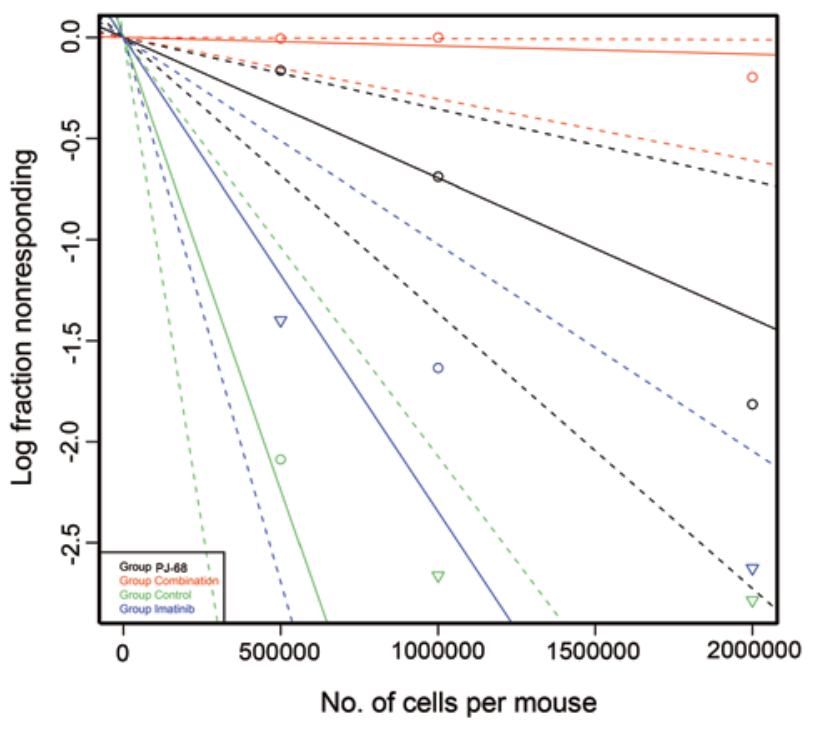

Control IM PJ-68 PJ-68+IM

Figure 7. Pharmacological inhibition of PRMT5 activity decreases the frequency of in vivo CML LSCs. (A) The mixture of $2 \times 10^{5} \mathrm{BM}$ cells from normal C57BL/6 mice with increasing numbers of CML BM cells $\left(2 \times 10^{6}, 1 \times 10^{6}\right.$, or $\left.5 \times 10^{5}\right)$ from CML mice receiving IM, PJ-68, or their combination $(n=8$ for each cell number) was transplanted into WT C57BL/6 mice irradiated at 550 cGy. Engraftment was monitored by analyzing peripheral blood (PB) every week, and GFP+ cells were detected in PB by flow cytometry. (B) The percentages of GFP+ cells at 16 weeks after transplantation. A percentage of GFP+ cells in PB greater than $0.5 \%$ was considered as positive engraftment. ${ }^{* *} P<0.0001$, 1-way ANOVA, post hoc intergroup comparisons, Tukey's test. Control ( $n=23$ ), IM ( $n=14)$, PJ-68 $(n=18)$, PJ-68+IM ( $n=22)$. (C) The frequency of LSCs after treatment is summarized in Table 1.

totally different from the previously reported PRMT5 inhibitors EPZ015666 and HLCL-61 (24, 54). PJ-68 effectively and selectively inhibited the methyltransferase activity of type II PRMT5 over type I PRMTs at nanomolar concentrations. Our PK studies showed that plasma concentrations (of about $3.57 \pm 0.08$ to $7.88 \pm$ $0.31 \mu \mathrm{M}$ ) of $\mathrm{PJ}-68$ are postulated to be sufficient to block in vitro histone methyltransferase activity of PRMT5. Our findings that PJ-68 decreased the in vitro and in vivo self-renewal capacity of CML LSCs indicate that further investigation of this lead compound and the therapeutic strategy of combining TKIs with relevant inhibitors of epigenetic modifiers is warranted.

\section{Methods}

\section{Compounds}

IM (imatinib, STI571) and the DNMT3A inhibitor SGI-1027 were purchased from Selleckchem. PJ-68 [N-((9-ethyl-9H-carbazol-3yl) methyl)-1,2,3,4-tetrahydronaphthalen-1-amine, Figure 2G] was obtained from Aurora Fine Chemicals and stored at $-20^{\circ} \mathrm{C}$ as a 100 $\mathrm{mM}$ stock solution in DMSO.

\section{Chemical reagents and DNA constructs}

DAPI was from Invitrogen. The TOPflash/FOPflash system and ChIP Kit were from Upstate Biotechnology. MG132, cycloheximide (CHX), propidium iodide (PI), human and murine PRMT5 shRNA, human BCR-ABL shRNA, human STAT5A, and STAT5B shRNA were from Sigma-Aldrich. pCMV-2B-Flag and pCMV-2B-Flag-PRMT5 constructs were provided by Shilai Bao (Institute of Genetics and Developmental Biology, Chinese Academy of Sciences, Beijing, China) (55); the MSCVBCR-ABL-IRES-EGFP construct was described previously (56); pcDNA3, pcDNA3-BCR-ABL, pSG5-BCR-ABL (p210), HA-tagged BCR, pSG5-ABL and pSG5-BCR-ABL (p190), lentiviral-TOP-dGFP-reporter, lentiviral-FOP-dGFP-reporter, pCMV-dR8.2, and pCMV-VSVG constructs were from Addgene.

\section{Cell lines}

The KBM5 and KBM5-T315I cells were from Sai-Ching J. Yeung (Department of Emergency Medicine, University of Texas M.D. Anderson Cancer Center, Houston, Texas, USA) and cultured in Iscove's modified Dulbecco's medium (Invitrogen) supplemented with 10\% FBS (57). K562 and M2-10B4 cells were purchased from 
A

$\mathrm{CML} \mathrm{CD} 34^{+}$cells

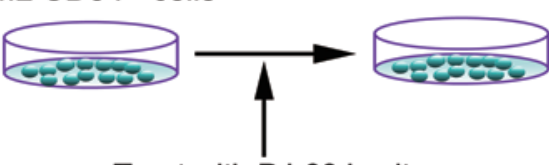

Treat with $\mathrm{PJ}-68$ in vitro

B

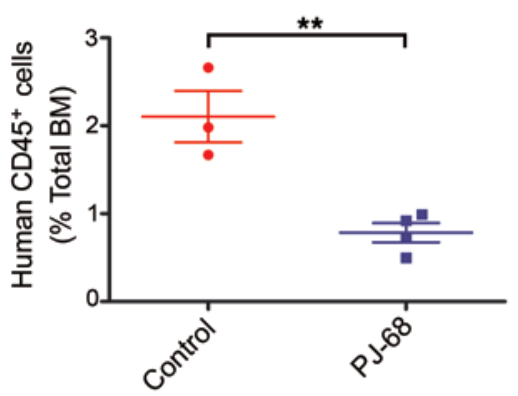

\section{$\mathbf{E}$}

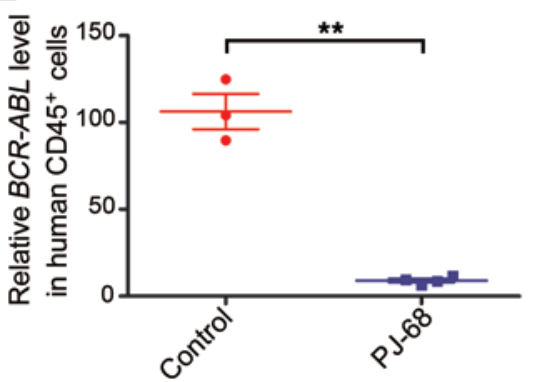

C

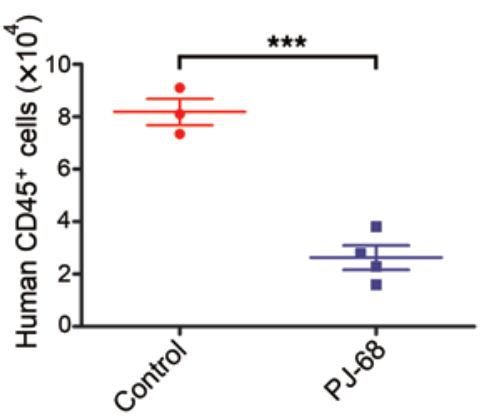

$\mathbf{F}$

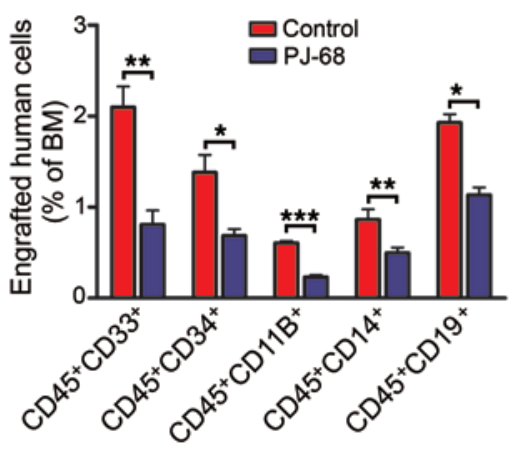

Figure 8. PRMT5 inhibition reduces long-term multilineage engraftment of human CML CD34+ cells in NSI mice. (A) Human CML CD34+ cells were treated with PJ-68 $(25.0 \mu \mathrm{M})$ in vitro for 3 days and injected into sublethally irradiated (300 cGy) NSI mice. After 12 weeks, human multilineage engraftment was analyzed by flow cytometry. (B and $\mathbf{C}$ ) The percentage (B) and the absolute number (C) of human $\mathrm{CD} 45^{+}$cells engrafted in the BM after transplantation of human CML CD34+ cells $\left(1 \times 10^{6}\right.$ cells/mouse) for 12 weeks. (D) The proportion of human CD45+ cells engrafted in the spleen at 12 weeks. (E) qRT-PCR analysis of $B C R-A B L$ mRNA level in $C D 45^{+}$cells engrafted in BM at 12 weeks.

(F) The proportion of engraftment of human CD33, CD34, CD11B, CD14, and CD19 in BM. (C) Representative flow cytometry plots of human CD45 and CD33 expression in mice with cells from 1 of the 2 CML patients ( $n=3$ for control, $n=4$ for PJ-68 treatment, each patient). ${ }^{*} P<0.05$, ${ }^{* *} P<0.01$,

${ }^{* *} P<0.0001,2$-tailed Student's $t$ test.

ATCC. Human uveal melanoma cells (Mel270) were provided by Martine Jager (Department of Ophthalmology, Leiden University Medical Center, Leiden, The Netherlands). K562, Mel270, and M2-10B4 cells were cultured in RPMI 1640 medium supplemented with $10 \%$ FBS (58). 293T and Plat-E cells were obtained from Cell Biolabs Inc. and maintained in DMEM with $10 \%$ FBS. Cells were kept at $37^{\circ} \mathrm{C}$ in a humidified incubator with $5 \% \mathrm{CO}_{2}$.

\section{Primary cells}

Peripheral blood or BM samples were obtained from patients with CML and from healthy adult donors in the First Affiliated Hospital of Sun Yat-sen University and Guangdong Provincial People's Hospital. The information for CML patients is in Supplemental Table 2. Mononuclear cells were isolated by Histopaque gradient centrifugation (density 1.077; Sigma-Aldrich) (59). CD34 ${ }^{+}$cells were isolated by use of the CD34 MicroBead Kit (Miltenyi Biotec). $\mathrm{CD} 34^{+} \mathrm{CD} 38^{-}$and $\mathrm{CD} 34^{+} \mathrm{CD} 38^{+}$cell populations were further sorted by flow cytometer (BD FACS Aria II) after staining with CD38PE antibody (catalog 130-098-907, Miltenyi Biotec). These primary cells were incubated in IMDM medium supplemented with $10 \%$ FBS in the presence of granulocyte-macrophage colony-stimulating factor (GM-CSF, $100 \mathrm{ng} / \mathrm{ml}$ ), stem cell factor (SCF, $100 \mathrm{ng} / \mathrm{ml}$ ), IL-3 (20 ng/ml), and IL-6 (20 ng/ml), which were purchased from PeproTech Inc. $(60,61)$.

\section{Measurement of intracellular PRMT5 and active $\beta$-catenin}

CML or NBM CD34 ${ }^{+}$cells or PJ-68-treated $\mathrm{K} 562$ cells were harvested, fixed in $4 \%$ formaldehyde for 10 minutes at $37^{\circ} \mathrm{C}$, and permea- 
A

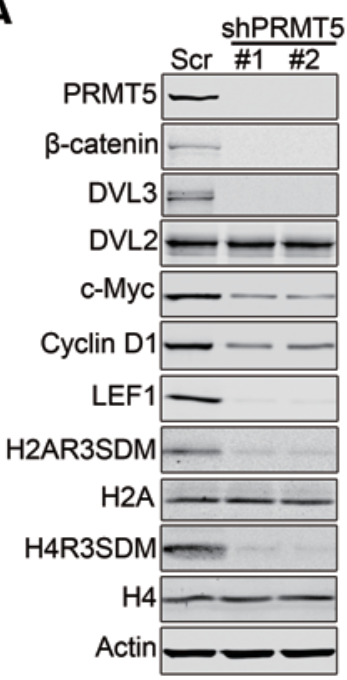

B

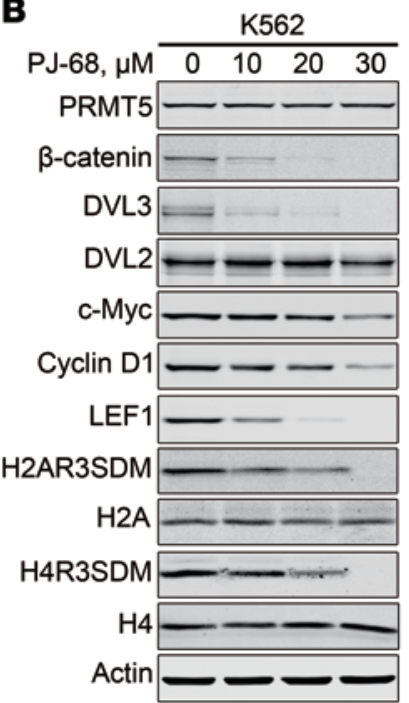

C

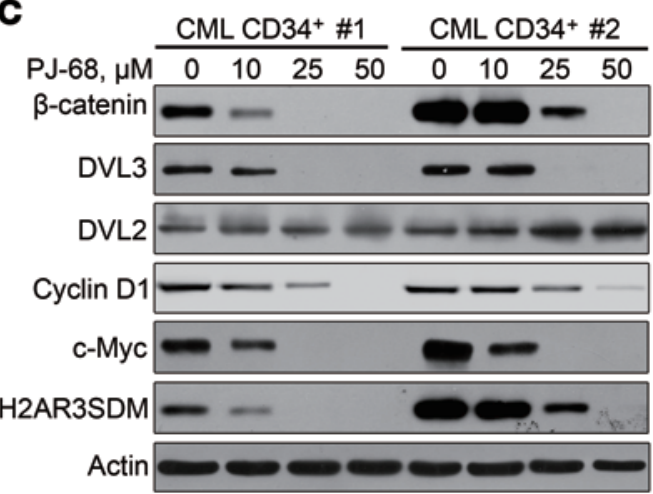

D

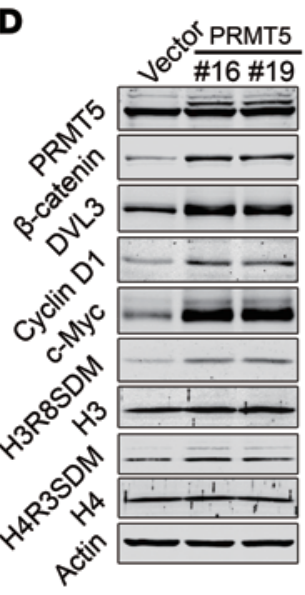

E

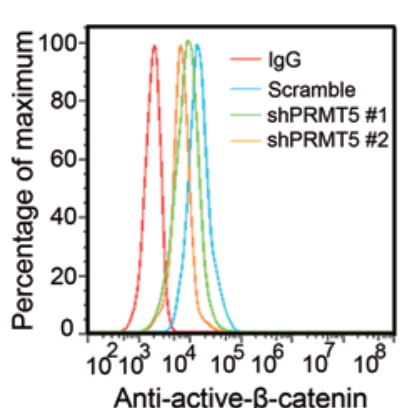

$\mathbf{F}$

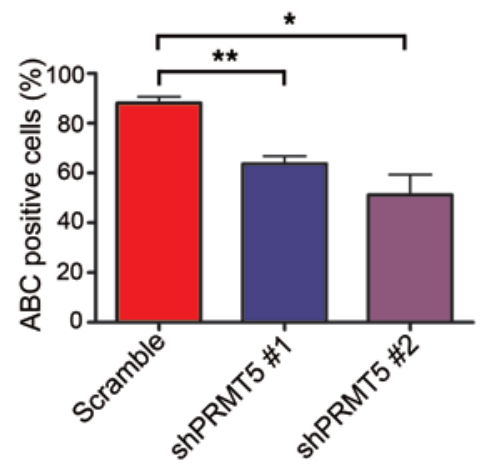

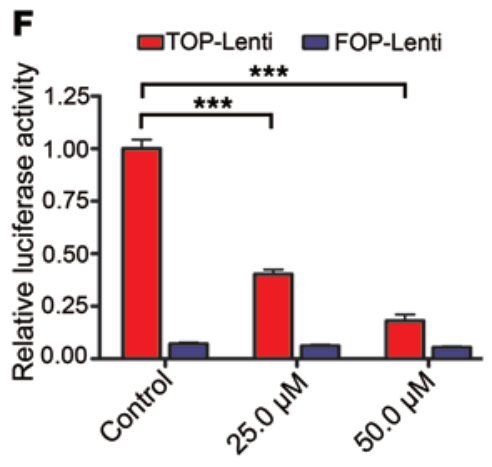

G

IB: $\beta$-catenin -
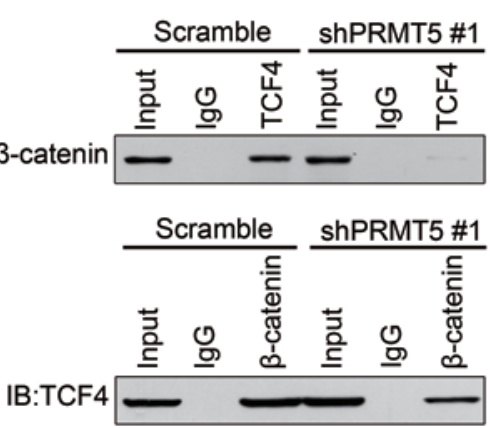

H

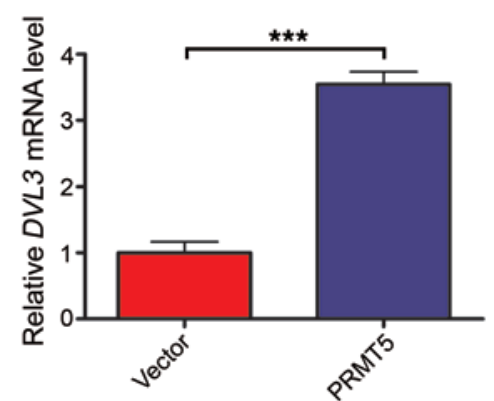

I

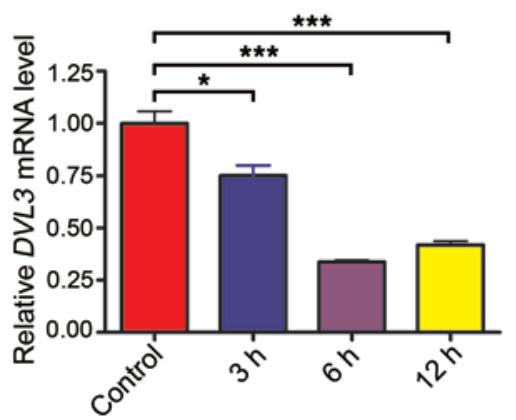

J

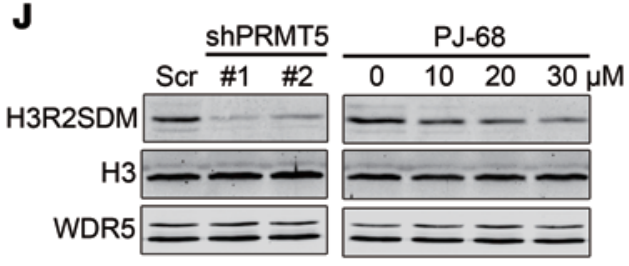

$\mathbf{K}$

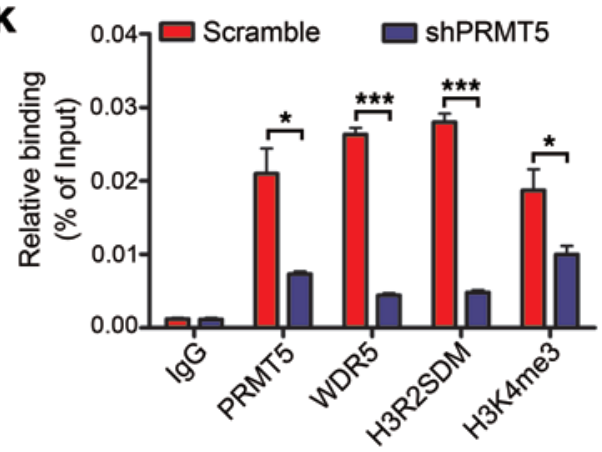


Figure 9. PRMT5 activates Wnt/ $\beta$-catenin signaling through upregulating DVL3 expression in human CML CD34+ cells. (A) PRMT5 knockdown by shRNA decreased the expression of Wnt/ $\beta$-catenin-related genes in CML cells. (B and C) Pharmacological abrogation of PRMT5 activity decreased the expression of $\beta$-catenin-related genes in $\mathrm{K} 562$ cells and in primary human CML CD34+ cells. (D) PRMT5 overexpression activated Wnt/ $\beta$-catenin signaling. (E) PRMT5 knockdown decreased active intracellular $\beta$-catenin levels in CML cells. Flow cytometry of activated intracellular $\beta$-catenin levels in $\mathrm{K} 562$ cells after labeling with anti-active $\beta$-catenin (ABC) antibody. Representative flow cytometry plot (left) and bar plots (right) are shown. (F) PRMT5 inhibition elicited TCF4/LEF1-dependent luciferase activity in CML CD34+ cells. Lenti, lentivirus. (G) PRMT5 knockdown disrupted the binding capability between $\beta$-catenin and TCF4. (H-K) PRMT5 activated Wnt/ $\beta$-catenin signaling through DVL3. (H) Ectopic PRMT5 expression increased DVL3 gene transcription. (I) PJ-68 inhibited transcription of the DVL3 gene. (J) PRMT5 knockdown or inhibition reduced the level of H3R2SDM as determined by Western blot analysis in K562 cells. (K) PRMT5 methylated histone H3R2 and recruited WDR5 to promote H3K4 trimethylation and DVL3 activation. ChIP experiments with antibodies specific for PRMT5, WDR5, H3R2SDM, and H3K4me3 showed significant enrichment of PRMT5 and its epigenetic marks at the DVL3 gene promoter, whereas knockdown of PRMT5 led to loss of recruitment of PRMT5 and its epigenetic marks on the DVL3 gene promoter in K562 cells. Two-tailed Student's $t$ test was performed for $\mathbf{F}, \mathbf{H}$, and $\mathbf{K}$; 1-way ANOVA, post hoc intergroup comparisons, Tukey's test was used for $\mathbf{E}$ and $\mathbf{I} .{ }^{*} P<0.05,{ }^{* *} P<0.01,{ }^{* *} P<0.0001$

bilized in prechilled $90 \%$ methanol for 30 minutes on ice (62). After washing and blocking, the cells were incubated with anti-PRMT5 or anti-active $\beta$-catenin, then stained with the secondary antibody Alexa Fluor 488-conjugated goat anti-rabbit or anti-mouse IgG. The FITC-conjugated mouse IgG1 $\kappa$ isotype control was stained similarly except for the secondary antibody. The samples were analyzed by flow cytometer (BD FACS Aria II).

\section{Western blot analysis}

The whole cell lysates were prepared in RIPA buffer (57, 61). Antibodies against c-ABL (C-19, catalog sc-887), H2A (H-124, sc-10807), H3 (FL-136, sc-10809), CRKL (C-20, sc-319), DVL3 (4D3, sc-8027),

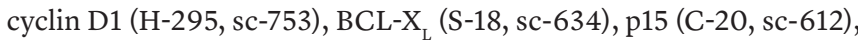
p27 (C-19, sc-528), and DNMT3A (H-295, sc-20703) were from Santa Cruz Biotechnology Inc. Antibodies against p-BCR-ABL (Y245) (catalog 2861), BCR (catalog 3902), p-CRKL (Y207) (catalog 3181), DVL2 (catalog 3224), LEF1 (C18A7, catalog 2286), and TCF4 (C48H11, catalog 2569) were from Cell Signaling Technology. Antibody against HA (16B12, catalog MMS-101P-200) was from Covance Inc. Antibodies against PRMT5 (catalog 07-405), H4 (clone 62-141-13, catalog 05-858), p-STAT5A/B (Y694/699) (catalog 07-586), STAT5A (catalog 06-553), STAT5B (catalog 06-969), p-STAT3 (Y705) (clone 9E12, catalog 05-485), STAT3 (catalog 06-596), active $\beta$-catenin (clone 8E7, catalog 05-665), p57 (catalog 06-556), H3R2SDM (catalog ABE460), H3K4me3 (catalog 07-473), and WDR5 (catalog 07-706) were from EMD Millipore. Antibodies against H4R3SDM (catalog ab5823), H3R8SDM (catalog ab130740), H2AR3SDM (catalog ab22397), and H4R3ADM (catalog ab194683) were from Abcam. Antibodies against $\beta$-catenin (clone $14 / \beta$-catenin, catalog 610153), c-Myc (catalog 551101), and p16 (catalog 551153) were from BD Biosciences. Antibodies against $\beta$-actin (clone AC-15, catalog A5441) and $\alpha$-tubulin (clone B-5-1-2, catalog T5168) were from Sigma-Aldrich. Detailed information about the secondary antibodies for Western blot analysis is shown in Supplemental Table 3. See the uncropped gels in Supplemental Figures 8-16.

\section{Lentiviral transduction in CML CD34+ cells}

Lentiviruses were produced by transient transfection in $293 \mathrm{~T}$ cells with control shRNA (Scramble) or specific shRNA together with the pCMVdR8.2 packing construct and the pCMV-VSVG envelope construct. CML or NBM CD $34^{+}$cells $\left(1 \times 10^{6}\right.$ cells $\left./ \mathrm{ml}\right)$ were infected by spinoculation $\left(1,500 \mathrm{~g}, 90\right.$ minutes, $\left.32^{\circ} \mathrm{C}\right)$ with virus-containing supernatants twice (63). Cells were harvested 48 hours later, and the knockdown effect was examined by Western blot analysis.

\section{Analysis of apoptosis of primary $\mathrm{CD} 34^{+}$cells}

CML CD $34^{+}$cells or NBM CD $34^{+}$cells were labeled with annexin V-FITC and CD38-PE antibodies after treatment; apoptotic cells $\left(\mathrm{CD} 34^{+} \mathrm{CD} 38^{-}\right.$annexin $\left.\mathrm{V}^{+}\right)$were detected by flow cytometer (BD FACS Aria II) (61).

CFSE assay. CFSE-labeled cells (CellTrace CFSE Proliferation Kit; Invitrogen) were cultured with PJ-68 for 96 hours, cells were harvested and stained with annexin V-PE (catalog 556422, BD Biosciences), and quiescent cell apoptosis $\left(\mathrm{CD} 34^{+} \mathrm{CD} 38^{-} \mathrm{CFSE}^{\max }\right.$ annexin $\left.\mathrm{V}^{+}\right)$was determined by flow cytometer (BD FACS Aria II) $(11,64,65)$.

\section{CFC/replating and LTC-IC assay}

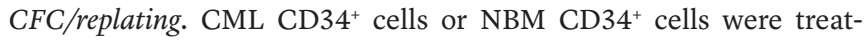
ed with different concentrations of PJ-68 for 24 hours, washed with PBS, and counted; 1,000 cells were plated in methylcellulose medium (MethoCult H4434, Stemcell Technologies). Colonies were counted on day 14. Cells were then harvested and counted, and 1,000 cells were replated for the secondary and tertiary rounds; colonies were counted on day 14 after each replating $(11,64,65)$.

LTC-IC assay. $2 \times 10^{6} \mathrm{CML}$ or NBM cells were seeded in LTC-IC medium onto irradiated (80 Gy) M2-10B4 cells and treated with PJ-68 $(25.0 \mu \mathrm{M})$ with or without IM $(2.5 \mu \mathrm{M})$ for 1 week; compound-containing medium was removed, and cells were cultured for 5 weeks in LTCIC medium (H5100, Stemcell Technologies) by weekly half-medium changes. After 6 weeks, cells were harvested and plated into methylcellulose medium (Methocult H4435, Stemcell Technologies) (11, 64, 65). LTC-IC-derived colonies were counted after 2 weeks.

\section{CML mouse model}

The retrovirus was produced by transient transfection with the MSCVBCR-ABL-IRES-EGFP construct in Plat-E cells. Viral supernatants were harvested at 48 and 72 hours after transfection and purified with the $0.45-\mu \mathrm{m}$ filter. BM cells from 6- to 8-week-old donor male C57BL/6 mice (Guangdong Medical Laboratory Animal Center) primed by 5-FU were stimulated with cytokines in vitro, then cells were transduced twice with the MSCV-BCR-ABL-IRES-EGFP retrovirus, followed by tail vein injection into irradiated (550 cGy) recipient female C57BL/6 mice. Following transplantation, mice were treated with $\mathrm{IM}(100 \mathrm{mg} / \mathrm{kg} / \mathrm{d}$, p.o.), PJ-68 (25 mg/kg/d and $50 \mathrm{mg} / \mathrm{kg} / \mathrm{d}$, i.p.), or both for 2 weeks; then mice were euthanized and $\mathrm{BM}$ and spleen cells were collected, labeled with the indicated antibodies, and analyzed by flow cytometer (BD FACS Aria II). BCR-ABL-expressing $\left(\mathrm{GFP}^{+}\right)$leukemia cells or myeloid cells were labeled with anti-mouse Gr-1-APC and Mac-1-PE antibodies (BD Pharmingen). Antibodies for lineage cocktail APC, Ly-6A/E 


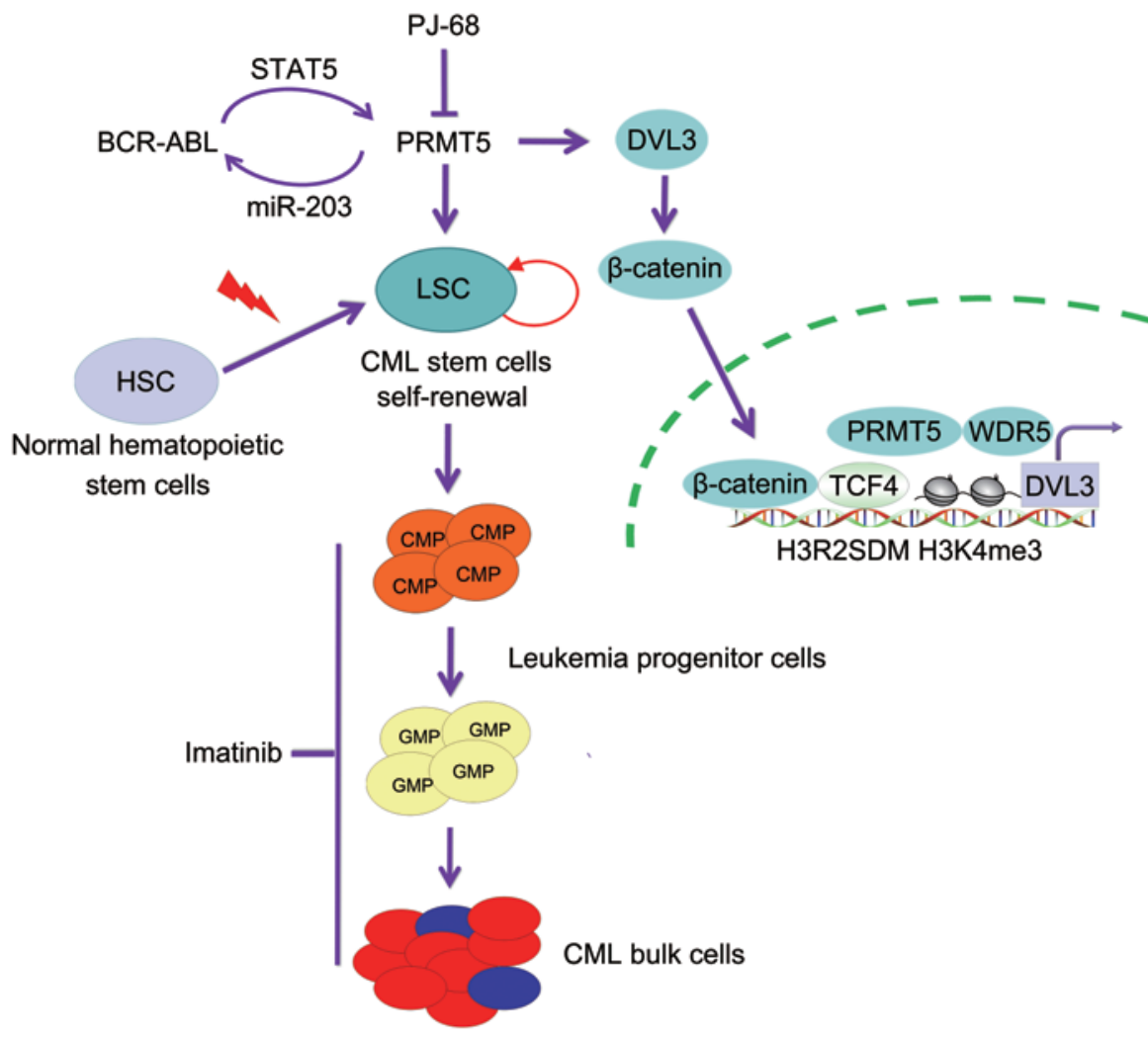

Figure 10. A proposed working model of a positive feedback loop between PRMT5 and BCR-ABL and targeting PRMT5 against self-renewal of CML LSCs. CML LSCs are insensitive to IM. There is a positive feedback loop between PRMT5 and BCR-ABL. STAT5depdendent PRMT5 expression is induced by $B C R-A B L$ during malignant transformation of normal HSCs. Increased PRMT5 expression reciprocally enhances $B C R-A B L$ gene expression via miR-203 in CML cells. PRMT5 is required for the survival and self-renewal of LSCs. Targeting PRMT5 in combination with IM eliminates LSCs in CML.

Leukemia blast

Progeny leukocyte

(Sca-1) PE-CF594, CD48 APC-Cy7, CD117 APC-H7, and CD34-PE (all mouse) were from BD Biosciences. Antibodies for CD117-PE, CD135 (Flt3) PE-Cy5, CD150 PE-Cy7, and CD16/CD32 (FcrRII/III) PE-Cy7 (all mouse) were from eBiocience. (The antibodies in this paragraph are detailed in Supplemental Table 4.) Granulocyte-macrophage pro-

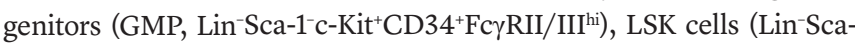
$1^{+} \mathrm{c}-\mathrm{Kit}^{+}$), LT-HSCs (LSK Flt3-CD150 ${ }^{+} \mathrm{CD} 48^{-}$), and ST-HSCs (LSK Flt3 $\left.\mathrm{CD}^{-} 0^{-} \mathrm{CD}^{-} 8^{-}\right)$in BM and spleen cells were detected $(11,12,64,65)$.

For evaluation of the in vivo effect of PRMT5 knockdown in murine CML LSK cells, LSK cells were sorted from the nucleated cells of BM and spleen of the primary-generation CML mice by flow cytometry and stimulated with cytokines in vitro overnight. They then underwent 2 rounds of transduction with lentiviral shRNA against murine PRMT5 for 48 hours and were subsequently transplanted into the secondary recipient C57BL/6 mice.

\section{qRT-PCR}

Total RNA was extracted by use of the RNeasy Mini Kit (QIAGEN). RNA concentrations were assessed using a NanoDrop ND-1000 spectrophotometer. Reverse transcription and qPCR were performed with a Maxima First Strand cDNA Synthesis Kit (Thermo Fisher Scientific) and SYBR Premix Ex Taq II (Takara Biomedical Technology). qRT-PCR assay was carried out on an Real-Time Thermocycler (CFX96, Bio-Rad) (62). The primers used are listed in Supplemental Table 5.

\section{Luciferase assay}

$293 T$ cells were transiently cotransfected with a $B C R-A B L$ promoter reporter construct $(0.5 \mu \mathrm{g})$ and vector, PRMT5, or PRMT5 (R368A) constructs, and
pEFRenilla-luc (10 ng) for 24 hours. K562 cells with Scramble, shPRMT5 \#1, or shPRMT5 \#2 were transfected with TOPflash or FOPflash constructs ( 0.5 $\mu \mathrm{g})$ and pEFRenilla-luc (10 ng) by electroporation for 24 hours. Luciferase activity was measured by use of a dual-luciferase assay kit (Promega) (61).

\section{ChIP}

ChIP was performed using the EZ-ChIP Kit (EMD Millipore) according to the manufacturer's instructions. K562 or shPRMT5 \#2 cells were crosslinked in $1 \%$ formaldehyde for 10 minutes at room temperature. Glycine was added to quench unreacted formaldehyde. The cells were washed with cold PBS, harvested, and resuspended in SDS lysis buffer containing a protease inhibitor cocktail. Cross-linked DNA was sheared to $200-1,000$ bp by sonication and centrifuged at $10,000 \mathrm{~g}$ at $4^{\circ} \mathrm{C}$ for 10 minutes. Protein $\mathrm{A} / \mathrm{G}$ agarose ( $60 \mu \mathrm{l}$ of a $50 \%$ slurry) was then added and incubated at $4^{\circ} \mathrm{C}$ for 1 hour. The supernatant was immunoprecipitated with anti-STAT5 antibodies (1:500) or normal rabbit IgG (negative control) at $4^{\circ} \mathrm{C}$ overnight. Pellets were washed, and protein-DNA complexes were eluted and reversed by overnight incubation at $65^{\circ} \mathrm{C}$ with proteinase $\mathrm{K}$. DNA was purified with spin columns. The purified DNA was used for PCR reaction. The PCR conditions were 5 minutes at $94^{\circ} \mathrm{C}$ for initial denaturation, 30 seconds at $94^{\circ} \mathrm{C}$ for denaturation, 30 seconds at $60^{\circ} \mathrm{C}$ for annealing, 30 seconds at $72^{\circ} \mathrm{C}$ for extension, repeat for total 35 cycles, $72^{\circ} \mathrm{C}$ for 5 minutes for final extension. qRT-PCR was performed as described previously (48). The primers for amplification of purified DNA are listed in Supplemental Table 5.

\section{Engraftment of human cells in immunodeficient mice}

CML CD $34^{+}$cells were cultured with PJ-68 $(25.0 \mu \mathrm{M})$ for 72 hours, and then cells $\left(1 \times 10^{6}\right.$ cells/mouse) were transplanted by tail vein injection 
into sublethally irradiated (300 cGy) 8-week-old NSI mice (Guangzhou Institutes of Biomedicine and Health, Chinese Academy of Sciences) (66). Mice were euthanized after 12 weeks, and BM cells and spleen cells were harvested; the engraftment of human cells was analyzed by flow cytometer $(11,12,64,65)$. Detailed information for generating NSI mice is described in the supplemental material. Detailed information about the antibodies for NSI mice is shown in Supplemental Table 6.

\section{Statistics}

All experiments were performed at least 3 times, and data represent mean \pm SD. GraphPad Prism 5.0 (GraphPad Software) was used for statistical analysis. Comparisons between 2 groups were analyzed by 2-tailed Student's $t$ test and comparisons of multiple groups by 1-way ANOVA, post hoc intergroup comparisons, and Tukey's test. A $P$ value less than 0.05 was considered statistically significant.

\section{Study approval}

This study was approved by the Sun Yat-sen University Ethics Committee according to institutional guidelines and Declaration of Helsinki principles, and written informed consent was received from participants prior to inclusion in the study. All animal studies were conducted with the approval of the Sun Yat-sen University Institutional Animal Care and Use Committee.

\section{Author contributions}

YLJ designed and performed experiments, analyzed data, and wrote the manuscript; FX performed the molecular docking exper- iments; BJ, JFZ, LJC, and YW performed experiments; JL and XD provided CML patient samples and clinical data; PL performed the NSI mouse experiments; RBR reviewed data; and JXP designed and guided research, analyzed data, and wrote the manuscript.

\section{Acknowledgments}

This study was supported by grants from the National Basic Research Program of China (973 Program grant no. 2009CB825506 to JXP), National Natural Science Funds (nos. 81025021, U1301226, 81373434, and 91213304 to JXP and no. 81473247 to YLJ), Guangdong Natural Science Funds for Distinguished Young Scholars (grant no. 2016A030306036 to YLJ), the Research Foundation of the Education Bureau of Guangdong Province, China (grant cxzd1103 to JXP), the Research Foundation of the Guangzhou Bureau of Science and Technology, Natural Science Foundation of the Guangdong province (grant 2015A030312014 to JXP), and the Fundamental Research Funds for the Central Universities (to JXP). The authors thank Sai-Ching J. Yeung (University of Texas M.D. Anderson Cancer Center) for a critical reading of the manuscript.

Address correspondence to: Jingxuan Pan, Jinan University Institute of Tumor Pharmacology, State Key Laboratory of Ophthalmology, Zhongshan Ophthalmic Center, Sun Yat-sen University, 54 South Xianlie Road, Guangzhou 510060, People's Republic of China. Phone: 86.20.37628262; E-mail: panjx2@ mail.sysu.edu.cn.
1. Druker BJ. Translation of the Philadelphia chromosome into therapy for CML. Blood. 2008;112(13):4808-4817.

2. Hochhaus A, et al. Favorable long-term follow-up results over 6 years for response, survival, and safety with imatinib mesylate therapy in chronic-phase chronic myeloid leukemia after failure of interferon-alpha treatment. Blood. 2008;111(3):1039-1043.

3. Ma L, et al. A therapeutically targetable mechanism of BCR-ABL-independent imatinib resistance in chronic myeloid leukemia. Sci Transl Med. 2014;6(252):252ra121.

4. Khorashad JS, et al. BCR-ABL1 compound mutations in tyrosine kinase inhibitor-resistant CML: frequency and clonal relationships. Blood. 2013;121(3):489-498.

5. Cortes JE, et al. A phase 2 trial of ponatinib in Philadelphia chromosome-positive leukemias. N Engl JMed. 2013;369(19):1783-1796.

6. Chan WW, et al. Conformational control inhibition of the BCR-ABL1 tyrosine kinase, including the gatekeeper T315I mutant, by the switch-control inhibitor DCC-2036. Cancer Cell. 2011;19(4):556-568.

7. Wu J, et al. Association between imatinib-resistant BCR-ABL mutation-negative leukemia and persistent activation of LYN kinase. J Natl Cancer Inst. 2008;100(13):926-939.

8. O'Hare T, Zabriskie MS, Eiring AM, Deininger MW. Pushing the limits of targeted therapy in chronic myeloid leukaemia. Nat Rev Cancer. 2012;12(8):513-526.

9. Sawyers CL. Shifting paradigms: the seeds of onco- gene addiction. Nat Med. 2009;15(10):1158-1161.

10. Carter BZ, Mak DH, Cortes J, Andreeff M. The elusive chronic myeloid leukemia stem cell: does it matter and how do we eliminate it? Semin Hematol. 2010;47(4):362-370.

11. Zhang B, et al. Effective targeting of quiescent chronic myelogenous leukemia stem cells by histone deacetylase inhibitors in combination with imatinib mesylate. Cancer Cell. 2010;17(5):427-442.

12. Zhang $\mathrm{H}$, et al. The Blk pathway functions as a tumor suppressor in chronic myeloid leukemia stem cells. Nat Genet. 2012;44(8):861-871.

13. Zhao C, et al. Loss of beta-catenin impairs the renewal of normal and CML stem cells in vivo. Cancer Cell. 2007;12(6):528-541.

14. Bhatia R, et al. Persistence of malignant hematopoietic progenitors in chronic myelogenous leukemia patients in complete cytogenetic remission following imatinib mesylate treatment. Blood. 2003;101(12):4701-4707.

15. Chen Y, Li S. Molecular signatures of chronic myeloid leukemia stem cells. Biomark Res. 2013;1(1):21.

16. Li L, et al. Activation of p53 by SIRT1 inhibition enhances elimination of CML leukemia stem cells in combination with imatinib. Cancer Cell. 2012;21(2):266-281.

17. Crews LA, Jamieson CH. Selective elimination of leukemia stem cells: hitting a moving target. Cancer Lett. 2013;338(1):15-22.

18. Konopleva M, et al. MEK inhibition enhances ABT-737-induced leukemia cell apoptosis via prevention of ERK-activated MCL-1 induction and modulation of MCL-1/BIM complex. Leukemia. 2012;26(4):778-787.

19. Zhang H, Li H, Ho N, Li D, Li S. Scd1 plays a tumor-suppressive role in survival of leukemia stem cells and the development of chronic myeloid leukemia. Mol Cell Biol. 2012;32(10):1776-1787.

20. Goff DJ, et al. A Pan-BCL2 inhibitor renders bone-marrow-resident human leukemia stem cells sensitive to tyrosine kinase inhibition. Cell Stem Cell. 2013;12(3):316-328.

21. Hou Z, et al. The LIM protein AJUBA recruits protein arginine methyltransferase 5 to mediate SNAIL-dependent transcriptional repression. Mol Cell Biol. 2008;28(10):3198-3207.

22. Yang Y, Bedford MT. Protein arginine methyltransferases and cancer. Nat Rev Cancer. 2013;13(1):37-50

23. Stopa N, Krebs JE, Shechter D. The PRMT5 arginine methyltransferase: many roles in development, cancer and beyond. Cell Mol Life Sci. 2015;72(11):2041-2059.

24. Tarighat SS, et al. The dual epigenetic role of PRMT5 in acute myeloid leukemia: gene activation and repression via histone arginine methylation. Leukemia. 2016;30(4):789-799.

25. Kryukov GV, et al. MTAP deletion confers enhanced dependency on the PRMT5 arginine methyltransferase in cancer cells. Science. 2016;351(6278):1214-1218.

26. Mavrakis KJ, et al. Disordered methionine metabolism in MTAP/CDKN2A-deleted cancers leads to dependence on PRMT5. Science. 2016;351(6278):1208-1213. 
27. Chittka A, Nitarska J, Grazini U, Richardson WD. Transcription factor positive regulatory domain 4 (PRDM4) recruits protein arginine methyltransferase 5 (PRMT5) to mediate histone arginine methylation and control neural stem cell proliferation and differentiation. J Biol Chem. 2012;287(51):42995-43006.

28. Tee WW, et al. Prmt5 is essential for early mouse development and acts in the cytoplasm to maintain ES cell pluripotency. Genes Dev. 2010;24(24):2772-2777.

29. Liu F, et al. Arginine methyltransferase PRMT5 is essential for sustaining normal adult hematopoiesis. J Clin Invest. 2015;125(9):3532-3544.

30. Liu F, et al. JAK2V617F-mediated phosphorylation of PRMT5 downregulates its methyltransferase activity and promotes myeloproliferation. Cancer Cell. 2011;19(2):283-294.

31. Pal S, Baiocchi RA, Byrd JC, Grever MR, Jacob ST, Sif S. Low levels of miR-92b/96 induce PRMT5 translation and H3R8/H4R3 methylation in mantle cell lymphoma. EMBO J. 2007;26(15):3558-3569.

32. Hoelbl A, et al. Stat5 is indispensable for the maintenance of bcr/abl-positive leukaemia. EMBO Mol Med. 2010;2(3):98-110.

33. Sun L, et al. Structural insights into protein arginine symmetric dimethylation by PRMT5. Proc Natl Acad Sci U S A. 2011;108(51):20538-20543.

34. Alinari L, et al. Selective inhibition of protein arginine methyltransferase 5 blocks initiation and maintenance of B-cell transformation. Blood. 2015;125(16):2530-2543.

35. Bueno MJ, et al. Genetic and epigenetic silencing of microRNA-203 enhances ABL1 and BCR-ABL1 oncogene expression. Cancer Cell. 2008;13(6):496-506.

36. Peng C, Li S. CML mouse model in translational research. Methods Mol Biol. 2010;602:253-266.

37. Sherr CJ, Roberts JM. CDK inhibitors: positive and negative regulators of G1-phase progression. Genes Dev. 1999;13(12):1501-1512.

38. Zhao Q, et al. PRMT5-mediated methylation of histone H4R3 recruits DNMT3A, coupling histone and DNA methylation in gene silencing. Nat Struct Mol Biol. 2009;16(3):304-311.

39. Clevers $H$, Nusse R. Wnt/ $\beta$-catenin signaling and disease. Cell. 2012;149(6):1192-1205.

40. Migliori V, et al. Symmetric dimethylation of H3R2 is a newly identified histone mark that supports euchromatin maintenance. Nat Struct Mol Biol. 2012;19(2):136-144.

41. Yuan CC, et al. Histone H3R2 symmetric dimethylation and histone $\mathrm{H} 3 \mathrm{~K} 4$ trimethylation are tightly correlated in eukaryotic genomes. Cell
Rep. 2012;1(2):83-90.

42. Coluccia AM, et al. Bcr-Abl stabilizes beta-catenin in chronic myeloid leukemia through its tyrosine phosphorylation. EMBO J. 2007;26(5):1456-1466.

43. Sattler M, Salgia R. Activation of hematopoietic growth factor signal transduction pathways by the human oncogene BCR/ABL. Cytokine Growth Factor Rev. 1997;8(1):63-79.

44. Iriyama N, et al. Shorter halving time of BCR-ABL1 transcripts is a novel predictor for achievement of molecular responses in newly diagnosed chronicphase chronic myeloid leukemia treated with dasatinib: results of the D-first study of Kanto CML study group. Am J Hematol. 2015;90(4):282-287.

45. Akita $\mathrm{H}$, et al. MYC activates stem-like cell potential in hepatocarcinoma by a p53-dependent mechanism. Cancer Res. 2014;74(20):5903-5913.

46. Casetti L, et al. Differential contributions of STAT5A and STAT5B to stress protection and tyrosine kinase inhibitor resistance of chronic myeloid leukemia stem/progenitor cells. Cancer Res. 2013;73(7):2052-2058.

47. Ye D, Wolff N, Li L, Zhang S, Ilaria RL. STAT5 signaling is required for the efficient induction and maintenance of CML in mice. Blood. 2006;107(12):4917-4925.

48. Yuan $\mathrm{H}$, et al. Activation of stress response gene SIRT1 by BCR-ABL promotes leukemogenesis. Blood. 2012;119(8):1904-1914.

49. Iwasaki M, Liedtke M, Gentles AJ, Cleary ML. CD93 marks a non-quiescent human leukemia stem cell population and is required for development of MLL-rearranged acute myeloid leukemia. Cell Stem Cell. 2015;17(4):412-421.

50. Notta F, et al. Evolution of human BCR-ABL1 lymphoblastic leukaemia-initiating cells. Nature. 2011;469(7330):362-367.

51. Chu I, et al. p27 phosphorylation by Src regulates inhibition of cyclin E-Cdk2. Cell. 2007;128(2):281-294.

52. Chakrabarti R, et al. $\Delta$ Np63 promotes stem cell activity in mammary gland development and basal-like breast cancer by enhancing Fzd7 expression and Wnt signalling. Nat Cell Biol. 2014;16(10):1004-1015

53. Ordóñez-Morán P, Dafflon C, Imajo M, Nishida E, Huelsken J. HOXA5 Counteracts stem cell traits by inhibiting Wnt signaling in colorectal cancer. Cancer Cell. 2015;28(6):815-829.

54. Chan-Penebre E, et al. A selective inhibitor of PRMT5 with in vivo and in vitro potency in MCL models. Nat Chem Biol. 2015;11(6):432-437.

55. Zhou Z, et al. PRMT5 regulates Golgi apparatus structure through methylation of the golgin
GM130. Cell Res. 2010;20(9):1023-1033.

56. Hao SX, Ren R. Expression of interferon consensus sequence binding protein (ICSBP) is downregulated in Bcr-Abl-induced murine chronic myelogenous leukemia-like disease, and forced coexpression of ICSBP inhibits Bcr-Abl-induced myeloproliferative disorder. Mol Cell Biol. 2000;20(4):1149-1161.

57. Wu Y, et al. Cyclin-dependent kinase 7/9 inhibitor SNS-032 abrogates FIP1-like-1 plateletderived growth factor receptor $\alpha$ and bcr-abl oncogene addiction in malignant hematologic cells. Clin Cancer Res. 2012;18(7):1966-1978.

58. Dai W, Zhou J, Jin B, Pan J. Class III-specific HDAC inhibitor Tenovin-6 induces apoptosis, suppresses migration and eliminates cancer stem cells in uveal melanoma. Sci Rep. 2016;6:22622.

59. Shi X, et al. Triptolide inhibits Bcr-Abl transcription and induces apoptosis in STI571resistant chronic myelogenous leukemia cells harboring T315I mutation. Clin Cancer Res. 2009;15(5):1686-1697.

60. Prosper F, Vanoverbeke K, Stroncek D, Verfaillie CM. Primitive long-term culture initiating cells (LTC-ICs) in granulocyte colony-stimulating factor mobilized peripheral blood progenitor cells have similar potential for ex vivo expansion as primitive LTC-ICs in steady state bone marrow. Blood.1997;89(11):3991-3997.

61. Jin Y, et al. Antineoplastic mechanisms of niclosamide in acute myelogenous leukemia stem cells: inactivation of the NF-kappaB pathway and generation of reactive oxygen species. Cancer Res. 2010;70(6):2516-2527.

62. Jin B, Ding K, Pan J. Ponatinib induces apoptosis in imatinib-resistant human mast cells by dephosphorylating mutant D816V KIT and silencing $\beta$-catenin signaling. Mol Cancer Ther. 2014;13(5):1217-1230

63. Neviani P, et al. PP2A-activating drugs selectively eradicate TKI-resistant chronic myeloid leukemic stem cells. JClin Invest. 2013;123(10):4144-4157.

64. Chu S, et al. Persistence of leukemia stem cells in chronic myelogenous leukemia patients in prolonged remission with imatinib treatment. Blood. 2011;118(20):5565-5572.

65. Neviani P, et al. FTY720, a new alternative for treating blast crisis chronic myelogenous leukemia and Philadelphia chromosome-positive acute lymphocytic leukemia. JClin Invest. 2007;117(9):2408-2421.

66. Xiao Y, et al. ANGPTL7 regulates the expansion and repopulation of human hematopoietic stem and progenitor cells. Haematologica. 2015;100(5):585-594. 